\title{
Recycling Cellular Energy for Self-Sustainable IoT Networks: A Spatiotemporal Study
}

\author{
Fatma Benkhelifa, Member, IEEE, Hesham ElSawy, Senior Member, IEEE, Julie A. McCann, Member, IEEE, \\ and Mohamed-Slim Alouini, Fellow, IEEE
}

\begin{abstract}
This paper investigates the self-sustainability of an overlay Internet of Things (IoT) network that relies on harvesting energy from a downlink cellular network. Using stochastic geometry and queueing theory, we develop a spatiotemporal model to derive the steady state distribution of the number of packets in the buffers and energy levels in the batteries of IoT devices given that the IoT and cellular communications are allocated disjoint spectrum. Particularly, each IoT device is modelled via a two-dimensional discrete-time Markov Chain (DTMC) that jointly tracks the evolution of the data buffer and energy battery. In this context, stochastic geometry is used to derive the energy generation at the batteries and the packet transmission success probability from buffers taking into account the mutual interference from other active IoT devices. To this end, we show the Pareto-Frontiers of the sustainability region, which define the network parameters that ensure stable network operation and finite packet delay. Furthermore, the spatially averaged network performance, in terms of transmission success probability, average queueing delay, and average queue size are investigated. For self-sustainable networks, the results quantify the required buffer size and packet delay, which are crucial for the design of IoT devices and time critical IoT applications.
\end{abstract}

Index Terms-Spatiotemporal models, stochastic geometry, queueing theory, energy harvesting, packet transmission success probability, two-dimensional discrete-time Markov chain, stability conditions.

\section{INTRODUCTION}

The Internet of Things (IoT) is the paradigm that bridges the physical and cyber worlds such that everything and anything will be connected to the Internet. Such ubiquitous and massive connectivity has unlimited potential to advance our life (e.g., smart cities, automated diagnostics, autonomous deriving, smart agriculture, public safety, etc.) [2]. Realizing the IoT paradigm brings potentially billions of new devices (e.g., sensors, actuators, machines, robots, vehicles, etc.) to the already congested wireless spectrum. In addition to the spectrum scarcity problem, IoT intrinsic features impose several new challenges to conventional wireless networks. For instance, IoT involves a multitude of heterogeneous devices with diverse

This work has been presented in part in [1] at IEEE Global Communications Conference (GLOBECOM'2018), Abu Dhabi, United Arab Emirates.

This work was funded in part by KAUST Office of Sponsored Research for the funding.

F. Benkhelifa and J.A. McCann are with the Imperial College London, London SW7 2AZ, United Kingdom (e-mail: \{f.benkhelifa, j.mccann\}@imperial.ac.uk).

H. ElSawy is with King Fahd University of Petroleum and Minerals (KFUPM), Dhahran, Saudi Arabia (e-mail: \{elsawyhesham\}@gmail.com).

M.S. Alouini is with King Abdullah University of Science and Technology (KAUST), Thuwal, Makkah Province, Saudi Arabia (e-mail: \{slim.alouini\}@kaust.edu.sa). quality of service requirements. Recharging/changing batteries of massive numbers of devices, that are distributed over a large scale area, represents another acute challenge for sustainable IoT operation. Hence, revolutionary wireless technologies and network designs are required to address the unprecedented challenges that arise in IoT networks [3], [4].

From the practical perspective, several new technologies and standards are evolving to cope with the surging IoT traffic. For instance, the recent 3rd Generation Partnership Project (3GPP) amendments propose Narrowband IoT (NB-IoT) and machine type communication (LTE-MTC) to accommodate IoT traffic within cellular networks [5]. Furthermore, several new low power wide area networks (LPWANs) are being developed and tailored for IoT use cases such as Sigfox, Weightless and LoRa [4], [6]. The 3GPP amendments and the LPWAN account for the intrinsic features of the IoT such as: massive density, sporadic traffic, multitude heterogeneity, and high-energy efficiency. At the device level, transceivers with energy harvesting capabilities are being developed to alleviate the administrative overhead of sustaining IoT networks [7].

In conjunction with the aforementioned industrial progress, the research community is developing rigorous mathematical techniques to characterize, design, and operate IoT networks. In this context, stochastic geometry (see [8] for a tutorial) is indispensable for characterizing the performance of interference limited IoT networks [9]-[11]. However, stand-alone stochastic geometry models fail to account for the sporadic traffic patterns of the IoT devices. Recently, several studies have integrated stochastic geometry and queueing theory to characterize the network performance while accounting for the spatiotemporal traffic generation. For instance, the work in [12], [13] characterizes the delay and the maximum spatiotemporal traffic that grant free and scheduled uplink cellular networks can accommodate. Random access for massive IoT uplink networks with unsaturated traffic is characterized in [14]. The work in [15], [16] studies the delay in downlink cellular networks with unsaturated traffic. The effect of different scheduling schemes on the delay in downlink cellular networks is studied in [17]. The work in [18] studies the percentile based performance (e.g., transmission success probability and delay) for a static ad hoc IoT network. However, the studies in [12][18] assume that all devices have perpetual energy sources. The problem of energy scarcity and harvesting for large-scale networks is considered in [19]-[22] via unsaturated energy queues. However, these studies [19]-[22], assume saturated data buffers for all devices. None of the aforementioned works consider unsaturated data buffers and unsaturated en- 
ergy queues to study the self-sustainability of large scale IoT networks. To the best of the authors knowledge, this paper is the first to characterize self-sustainability frontiers, delay, coverage probability in a large scale IoT network that is powered by recycling ambient energy.

This paper proposes a novel spatiotemporal mathematical model, based on stochastic geometry and queueing theory, to characterize and design self-sustainable IoT networks. We consider an IoT network modeled via a Poisson bipolar process (PBP), where each device is equipped with a data buffer and an energy queue (i.e., battery). Data is generated, and stored for transmission, at each buffer according to an independent and identically distributed (i.i.d.) geometric distribution. Batteries are replenished by scavenging radio frequency (RF)-energy from a downlink cellular network, where the base-stations (BSs) are modeled via an independent Poisson point process (PPP). A transmission attempt occurs from an IoT device when the data buffer is non-empty and the energy stored in the battery is sufficient for transmission. The transmission attempt is successful if the signal-to-interference ratio (SIR) at the receiver is above a certain threshold. ${ }^{1}$ Hence, each bipolar link is modeled via a two-dimensional discrete-time Markov Chain (DTMC) and the IoT network is considered as a network of spatially interacting DTMCs. To this end, we obtain the packet transmission success probability at the network steady state and characterize the self-sustainability region of the network. ${ }^{2}$ The main contributions of this paper are summarized below:

- To the best of our knowledge, this paper presents the first mathematical model that jointly accounts for spatiotemporal traffic generation, energy harvesting problem, and mutual interference between devices in a large scale IoT network. A novel spatiotemporal model is developed to account for the unsaturated batteries and data buffers for IoT networks powered by cellular downlink energy.

- A novel two dimensional quasi birth death DTMC is developed to jointly track energy generation/depletion along with packet generation/transmission in the IoT devices. While the packet generation is assumed to be geometric, energy generation and successful packet transmissions are model via a phase type (PH) distribution that accounts for, respectively, energy harvesting from the downlink cellular network and mutual interference among the IoT devices.

- We show the Pareto-frontiers of the sustainability region, which characterize the maximum spatiotemporal traffic that an IoT network can accommodate via recycling the RF-power of a cellular network with a given BS density. We also study the performance metrics such as the transmission success probability, the average queue size, and the average queueing delay.

- We illustrate the spectrum scarcity and energy scarcity tradeoff within self-sustainable IoT networks.

\footnotetext{
${ }^{1}$ The scarcity of the wireless spectrum along with the massive density of devices leads to aggressive spatial frequency reuse. Consequently, the aggregate interference dominates the thermal noise effect and becomes the performance limiting parameter

${ }^{2}$ The self-sustainability region defines all network parameters that ensure stable data buffers and finite transmission delay across the network.
}

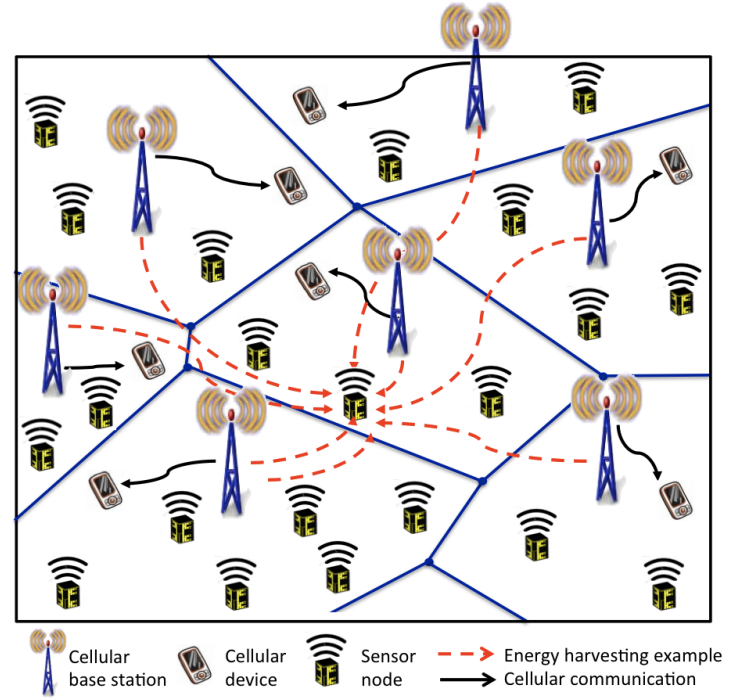

Figure 1. Downlink cellular network with BSs communicating with cellular users, and EH-enabled IoT transmitters communicating with intended IoT receivers.

\section{System Model}

For the sake of organized treatise, we discuss the spatial, temporal, and energy harvesting models in separate sections.

\section{A. Spatial and Propagation Models}

We consider an IoT network that is spatially distributed in $\mathbb{R}^{2}$ according to a homogeneous PBP with spatial intensity $\lambda_{D}$. That is, the IoT network is constituted from IoT transmitterreceiver pairs, denoted as bipolar links, where each link has a fixed length of $r_{0}$ and a uniform random orientation $\varepsilon \in$ $[0,2 \pi]$. According to the PBP, the IoT transmitting devices constitute a PPP, denoted as $\Phi_{D}=\left\{y_{j}: j=1,2, \ldots\right\}$, where $y_{j} \in \mathbb{R}^{2}$ denotes the location of the $j$ 'th IoT transmitter. ${ }^{3}$ The IoT network coexists with a cellular downlink network that consists of BSs that are spatially distributed according to an independent PPP, denoted as $\Phi_{B}=\left\{z_{i}: i=1,2, \ldots\right\}$, with spatial intensity $\lambda_{B}$, where $z_{i} \in \mathbb{R}^{2}$ denotes the location of the $i$ 'th cellular BS.

We consider a Rayleigh fading environment with i.i.d. unit variance channels. The channel gains between the BSs and the IoT devices are denoted by $h$ and the channel gains between IoT devices are denoted by $g$. All channels gains are assumed to be independent from each other as well as from the spatial locations. We utilize a power-law path loss model such that the signal power decays at the rate $r^{-\alpha}$, where $r$ is the propagation distance and $\alpha$ is the path loss exponent. ${ }^{4}$ The path loss exponent between IoT devices is denoted as $\alpha_{D}$, which is generally different from the path loss exponent between a BS and an IoT device, denoted as $\alpha_{B}$.

${ }^{3}$ For bidirectional communications, the D2D transmitter and receiver within the same D2D link can exchange roles. By virtue of the displacement theorem of PPPs, the same analysis applies for either the forward or reverse links.

${ }^{4}$ The utilized unbounded path loss model is verified in [23] for the aggregate network power received at a typical point for path loss exponents less than or equal to 4 . 
Each BS becomes active with probability $b \in[0,1]$ independently of other BSs, where active BSs transmit with a fixed power $P_{B}{ }^{5}$ The IoT network operates at a dedicated spectrum that is universally reused by all the IoT devices (e.g., LTE direct). Hence, the IoT devices mutually interfere with each other but there is no interference between the cellular and IoT networks. All IoT devices randomly and uniformly access 1 out of $N_{c}$ channels. Each IoT transmitter inverts its path-loss towards its associated receiver such that the useful average received power at each IoT receiver is maintained at a known value $\rho_{D}$ [26]. Since the distance between the IoT transmitters and their intended IoT receivers is fixed, each IoT transmitter requires a transmit power $P_{D}$ to transmit one packet such that $P_{D}$ is given by $P_{D}=\rho_{D} r_{0}^{\alpha_{D}}$.

\section{B. Temporal and Queueing Model}

We consider a discrete-time slotted system with slot duration of $T_{s}$ seconds. Each IoT transmitter is equipped with a data buffer. The data packets are generated at each IoT device according to an i.i.d. geometric distribution with parameter $a \in[0,1]$. For each device, the generated packets are stored in the data buffer to be transmitted over the wireless channel to the intended receiver according to the first-in first-out (FIFO) discipline. Packets are transmitted one-byone, where a transmission attempt takes place if and only if, there is sufficient energy in the device battery for path loss inversion and the data buffer has at least one packet. Due to the mutual interference between devices, a packet transmission fails if the SIR at the receiver is less than a predetermined threshold $\theta$. If a transmission failure occurs, the failed packet will be persistently retransmitted in the following transmission attempts until successful transmission. ${ }^{6}$ Only packets that are successfully transmitted to the intended receiver are discarded from the buffer.

\section{Energy Harvesting Model}

All IoT transmitters rely on scavenging the downlink RFenergy from the cellular network to replenish their batteries. Hence, each IoT transmitter is equipped with an energy receiver and rechargeable battery (i.e., energy queue) for energy harvesting with capacity $B$. The energy receiver is tuned to the downlink cellular frequency, which is different from the IoT dedicated frequency. As such, the IoT transmitters only harvest from the cellular network downlink power.

The IoT transmitters employ the well-known "harvest-thentransmit" protocol, which is suited for IoT devices due to its simplicity. All IoT devices are equipped with a single antenna switching between the energy receiver and information transmitter. Due to channel inversion power control, the sufficient energy to invert the path loss towards the intended receiver is $P_{D}=\rho_{d} r_{0}^{\alpha_{D}}$. Hence, each packet transmission attempts

\footnotetext{
${ }^{5}$ The activity factor $b$ reflects the time-varying user loads and traffic per BS [24], [25].

${ }^{6}$ Different from conventional collision models, the considered D2D network has several sources of randomization (e.g., fading, EH, and channel access) to resolve conflicts in packet retransmissions.
}

deducts $P_{D}$ from the device's battery. ${ }^{7}$ It is assumed that the full energy capacity of the battery at each IoT device is sufficient for transmitting $N$ packets, i.e. $B=N P_{D}$. In this paper, we assume that information transmission is prioritized over energy harvesting. Hence, all transmitters with nonempty data buffers and sufficient energy in their batteries are in the information transmission mode. Devices with nonempty buffers and insufficient energy in their batteries will persistently harvest energy, in the energy reception model, until the threshold $P_{D}$ is achieved. Once the battery level $P_{D}$ is achieved, the transmitter switches to the information transmission mode in the subsequent time slot. Only devices with empty buffers can persistently harvest energy until the battery is full. Note that any packet arrival, to a device with empty buffer and sufficient energy in its battery, will interrupt the energy harvesting and switch the device to the information transmission mode. We denote the transmutation activity probability, i.e., the probability of having non-empty data buffer and sufficient energy for transmission (at least $P_{D}$ ), with $d$.

\section{Methodology of Analysis}

In many IoT use cases (e.g., smart parking meters), the IoT devices are fixed. Furthermore, in nomadic and low-mobility IoT scenarios, the fading and device/BS activity vary at a much shorter time scale compared to the time required to make tangible location displacement for IoT devices. Hence, it is reasonable to assume an arbitrary, but static, cellular and IoT networks where only fading and devices/BSs activity change over time. In such a static network setting, each IoT device may have its own location dependent performance [18], [27], [28] in terms of harvesting and transmission success probability. However, the activity probabilities $b$ and $d$ mitigate such location dependent discrepancies (i.e., decrease the variance) across the IoT devices performance [12], [27]. Exploiting this fact, we use the following approximation:

Approximation 1: The spatially averaged harvesting probabilities and transmission success probability of a typical IoT device is representative of all IoT devices in the network.

Remark 1: The effect of Approximation 1 diminishes as $b$ and $d$ decrease as different realization of, respectively, active BSs from $\Phi_{B}$ and active devices from $\Phi_{D}$ appear in each time slot. Note that the foreseen ultra-densification of cellular networks will dramatically decrease the load served by each BS and will lead to small activity factor $b$. Furthermore, $d$ is already small in the depicted network model due to the sporadic pattern of the IoT traffic along with the employed "harvestthen-transmit" scheme. Note that the analysis becomes exact in high mobility scenarios [28].

Remark 2: The cellular network is not utilized for information transfer within the IoT network and is used for energy harvesting only. Hence, the aggregate downlink power at each time slot comes from the independently thinned PPP $\Phi_{B}$, with

\footnotetext{
${ }^{7}$ Other energy consumption sources (e.g., circuit power) and losses (e.g., mismatch losses) can be incorporated by defining the transmission threshold as $\rho_{d} r_{0}^{\alpha_{D}}+C$, where $C$ is the energy required to power the circuits and for signal processing.
} 
intensity $b \lambda_{B}$, and the nearest BS to the IoT device is not necessarily contributing to the harvested energy. Hence, the energy harvesting variance among the devices does not saturate when decreasing $b$ as in the case of success probability in downlink information transfer shown in [27].

Remark 3: The analysis in this paper can be extended to the case of static network with high $b$ and/or $d$ by following the same methodology in [18], which is postponed to future extension.

For simplicity, we discretize the battery into $N \times M$ energy levels of equal amount $\omega=\frac{B}{N M}=\frac{P_{D}}{M}$. Let $\boldsymbol{p}=\left[p_{0}, p_{m}, \cdots, p_{N M}\right]$, where $p_{m}, 0 \leq m \leq N M$, is the spatially averaged probability that the harvested energy is sufficient for replenishing $m$ battery levels in one time slot. Furthermore, let $p_{c}$ denote the spatially averaged probability of successful packet transmission and let $(\cdot)=(1-\cdot)$ denote the probability complement operator. Exploiting Approximation 1, the microscopic (i.e., individual) behavior of any IoT device can be modeled via the two-dimensional DTMC shown in Fig. 2 to track the temporal evolution of the packets in the buffer (levels) and energy in the battery (phases). As shown in Fig. 2, the battery may recharge $m$ phases per time slot with probability $p_{m}$. On the other hand, a single-step transition can occur between data buffer levels as only one packet can be generated and/or transmitted per time slot. Note that a packet departure can only take place from the phases greater than or equal to $M$ such that the stored energy is sufficient for at least one transmission attempt. After each transmission attempt, the battery is depleted and the DTMC goes down with $M$ phases. Another transmission attempt happens if the remaining energy is greater or equal to $P_{D}$ and the data buffer is nonempty. Otherwise, the DTMC resets the energy harvesting process. Note that while all IoT devices are represented via the same DTMC model, different IoT devices can have different buffer and battery states at a given time slot.

While the DTMC in Fig. 2 describes the microscopic behavior of an IoT device, the parameters $\boldsymbol{p}$ and $p_{c}$ are functions of the macroscopic (i.e., network wide) mutual interactions among all IoT devices and BSs. Particularly, the harvesting probability vector $\boldsymbol{p}$ is determined according to the distribution of the aggregate downlink power at the IoT transmitter from all active BSs. Furthermore, the transmission success probability $p_{c}$ is function of the interference power received by the IoT receiver from all other active IoT transmitters. Hence, stochastic geometry is employed to find the parameters $\boldsymbol{p}$ and $p_{c}$ in Section III-A and queueing theory is utilized to find the steady state distribution vector of the DTMC in Fig. 2 in Section III-B. Note that the stochastic geometry analysis and queueing theory analysis are interdependent because i) finding $p_{c}$ requires the intensity of mutually interfering IoT devices $d \lambda_{D}$; and ii) obtaining $d$ (i.e., from steady state probability of the DTMC) requires $p_{c}$. Such interdependence is solved via an iterative solution in Section III-C based on the fixed point theorem.

\section{Spatiotemporal Model}

\section{A. Stochastic Geometry Analysis}

In this section, we use stochastic geometry to derive the spatially averaged transmission success probability $p_{c}$ and the energy harvesting probability vector $\boldsymbol{p}$. Without loss of generality, we focus on a test device located at an arbitrary origin, which becomes the typical device under spatial averaging.

1) Packet Transmission: Let $\tilde{\Phi}_{D}$ be the point process of mutually interfering IoT devices and let the IoT receiver of the test bipolar IoT link be located at an arbitrary origin. Conditioned that a transmission attempt at the test link took place, a successful packet delivery occurs if the SIR at the test receiver is above a certain detection threshold $\theta$. Hence, the transmission success probability is given by

$$
p_{c}(\theta)=\mathbb{P}\left\{\frac{\rho_{D} g_{0}}{P_{D} \sum_{y_{j} \in \tilde{\Phi}_{D} \backslash y_{0}} g_{j}\left\|y_{j}\right\|^{-\alpha_{D}}}>\theta\right\},
$$

where $\|\cdot\|$ is the Euclidean norm. The transmission success probability is characterized via the following theorem.

Theorem 1: The packet transmission success probability for a typical IoT link is given by

$$
p_{c}(\theta)=\exp \left(-\frac{2 \pi^{2} d \lambda_{D} r_{0}^{2}}{\alpha_{D} \sin \left(\frac{2 \pi}{\alpha_{D}}\right)} \theta^{\frac{2}{\alpha_{D}}}\right) .
$$

Proof: The transmission success probability in (1) is expressed, using the complementary density function (CDF) of the exponential distribution with unit mean, as

$$
p_{c}(\theta)=\mathbb{E}_{y_{j}, g_{j}}\left[\exp \left(-\frac{\theta}{\rho_{D}} P_{D} \sum_{y_{j} \in \tilde{\Phi}_{D} \backslash y_{0}} g_{j}\left\|y_{j}\right\|^{-\alpha_{D}}\right)\right]=\mathcal{L}_{D}\left(\frac{\theta}{\rho_{D}}\right),
$$

where $\mathcal{L}_{D}(\cdot)$ is the Laplace transform (LT) of the aggregate interference derived in (40) in Appendix A, which proves Theorem 1.

If the fixed distance $r_{0}$ assumption is relaxed by assuming a density function for the distance $r_{0}$, we have the following corollary.

Corollary 1: If the distance $r_{0}$ between the D2D transmitter and its dedicated receiver is random according to a certain PDF $f_{r_{0}}(\cdot)$, the packet transmission success probability becomes

$$
p_{c}(\theta)=\mathbb{E}_{r_{d}}\left[\exp \left(-\frac{2 \pi^{2} d \lambda_{D}}{\alpha_{D} \sin \left(\frac{2 \pi}{\alpha_{D}}\right)} \theta^{\frac{2}{\alpha_{d}}} r_{d}^{2}\right)\right] .
$$

Proof: The proof follows the same steps as in Appendix A.

Proposition 1: When the receiver is uniformly distributed in a circle of radius $R_{c}$ around the transmitter, i.e. $f_{r_{d}}(r)=\frac{2 r}{R_{c}^{2}}$ with $0 \leq r \leq R_{c}$, the success transmission probability is given by

$$
p_{c}(\theta)=\frac{1-\exp \left(-\frac{2 \pi^{2} d \lambda_{D}}{\alpha_{D} \sin \left(\frac{2 \pi}{\alpha_{D}}\right)} \theta^{\frac{2}{\alpha_{d}}} R_{c}^{2}\right)}{\frac{2 \pi^{2} d \lambda_{D}}{\alpha_{D} \sin \left(\frac{2 \pi}{\alpha_{D}}\right)} \theta^{\frac{2}{\alpha_{d}}} R_{c}^{2}} .
$$

Proof: The proof is a direct application of Corollary 1. 


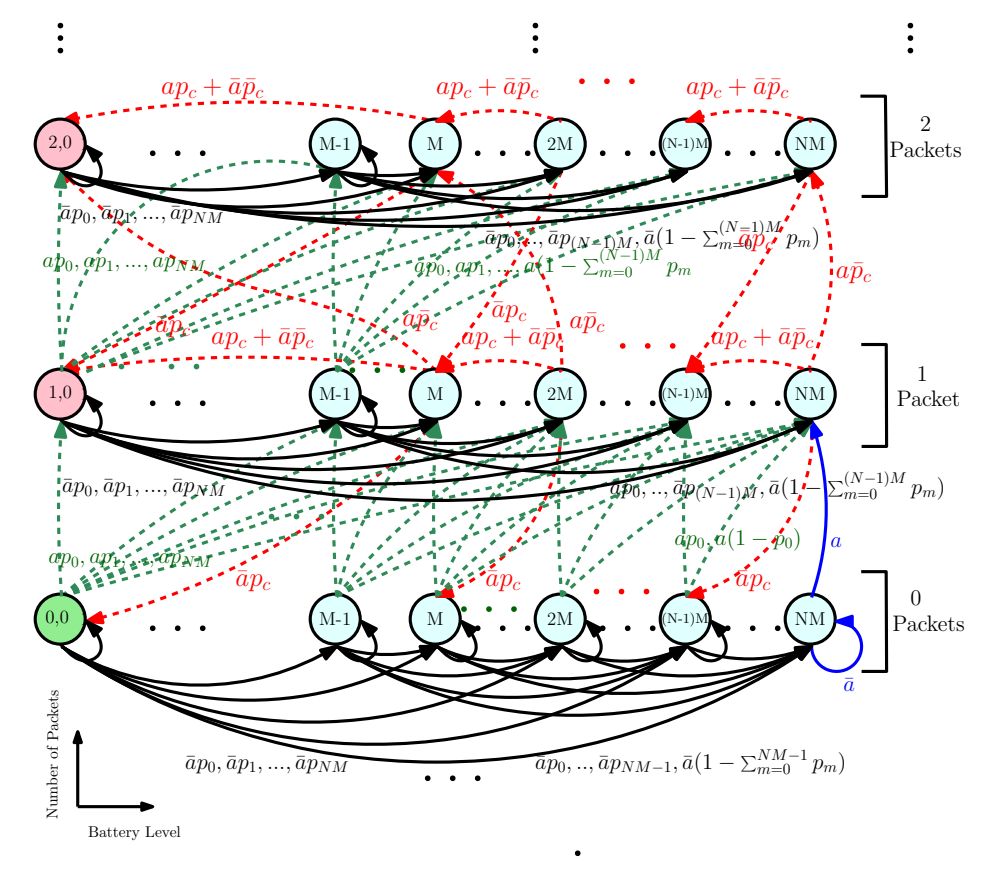

Figure 2. Markov chain diagram

Note that, for the random $r_{0}$, we still have to harvest an amount of power $P_{D}$, but the received power is no more $\rho_{D}$, but another value equal to $P_{D} r_{0}^{-\alpha_{D}}$.

2) Energy Harvesting: For a given time slot, let $\tilde{\Phi}_{B}$ be the point process of simultaneously active BSs. We denote by $P_{r}=P_{B} \sum_{z_{i} \in \tilde{\Phi}_{B}} h_{i}\left\|z_{i}\right\|^{-\alpha_{B}}$ the received power at the test IoT transmitter at the origin. The CDF of the received power $P_{r}$ is characterized in the following theorem:

Theorem 2: The CDF of the received power in a generic time slot is given by

$$
\begin{aligned}
& F_{P_{r}}(y)=1-\frac{\alpha_{B}}{2 \pi} \int_{0}^{\infty} \frac{\exp \left(-y\left(\frac{t}{\kappa_{0}}\right)^{\frac{\alpha_{B}}{2}}-t \cos \left(\frac{2 \pi}{\alpha_{B}}\right)\right) \sin \left(t \sin \left(\frac{2 \pi}{\alpha_{B}}\right)\right)}{t} d t \\
& \stackrel{\left(\alpha_{B}=4\right)}{=} \operatorname{erfc}\left(\frac{\pi^{2} \delta_{0}}{4 \sqrt{y}}\right),
\end{aligned}
$$

where $\kappa_{0}=\frac{2 \pi^{2} b \lambda_{B} P_{B}^{\frac{2}{\alpha_{B}}}}{\alpha_{B} \sin \left(\frac{2 \pi}{\alpha_{B}}\right)}, \delta_{0}=b \lambda_{B} \sqrt{P_{B}}, \operatorname{erf}(z)=\frac{2}{\sqrt{\pi}} \int_{0}^{z} e^{-t^{2}} d t$ is the error function and $\operatorname{erfc}(\cdot)=1-\operatorname{erf}(\cdot)$ is the complementary error function. For $\alpha_{B}=4$, the probability density function (PDF) of $P_{H}$ is expressed as

$$
f_{P_{r}}(y)=\frac{\delta_{0}}{4}\left(\frac{\pi}{y}\right)^{3 / 2} \exp \left(-\frac{\left(\frac{\pi}{2}\right)^{4} \delta_{0}^{2}}{y}\right) .
$$

Proof: See Appendix B.

Most research works consider a linear energy harvesting model to describe the relationship between the received power $P_{r}$ and the harvested power $P_{H}$ at the test IoT transmitter. However, the linear model is accurate only for low powers where the practical energy harvesting model is approximated by the linear model [29], [30]. For higher powers, the linear model is no more accurate in practice. For moderate to high powers, the practical energy harvesting model is approximated by the nonlinear model until it saturates at a certain point [30]. There are different nonlinear energy harvesting models in the literature: sigmoidal model [31], multi-sine model [32], heuristic model [33], etc. To generalize our energy harvesting model and take into consideration either linearity or nonlinearity, we consider a general function $\Psi(\cdot)$ modelling the relationship between $P_{r}$ and $P_{H}$ at each IoT device as

$$
P_{H}=\Psi\left(P_{r}\right)=\Psi\left(P_{B} \sum_{z_{i} \in \tilde{\Phi}_{B}} h_{i}\left\|z_{i}\right\|^{-\alpha_{B}}\right) .
$$

The function $\Psi(\cdot)$ is continuous differentiable and can be either linear (i.e. $\Psi(x)=\zeta x$, where $\zeta \in[0,1]$ is the harvesting efficiency) or nonlinear and has a couple of properties, for $x \geq 0$, such as

$$
0 \leq \Psi(x) \leq x, \quad \text { and } \quad \Psi^{-1}(x) \neq 0 .
$$

Following that, the CDF of the harvested power $P_{H}$ is given in the following proposition:

Proposition 2: Given that $\Psi(\cdot)$ is continuous, differentiable and invertible, the CDF of the harvested power in a generic time slot is given in the following proposition

$$
\begin{aligned}
& F_{P_{H}}(x)=F_{P_{r}}\left(\Psi^{-1}(x)\right) \\
& =1-\frac{\alpha_{B}}{2 \pi} \int_{0}^{\infty} \frac{\exp \left(-\Psi^{-1}(x)\left(\frac{t}{\kappa_{0}}\right)^{\frac{\alpha_{B}}{2}}-t \cos \left(\frac{2 \pi}{\alpha_{B}}\right)\right)}{t} \sin \left(t \sin \left(\frac{2 \pi}{\alpha_{B}}\right)\right) d t
\end{aligned}
$$

$$
\stackrel{\left(\alpha_{B}=4\right)}{=} \operatorname{erfc}\left(\frac{\pi^{2} \delta_{0}}{4 \sqrt{\Psi^{-1}(x)}}\right) .
$$

For $\alpha_{B}=4$, the probability density function (PDF) of $P_{H}$ is expressed as

$$
f_{P_{H}}(x)=\left(\Psi^{-1}(x)\right)^{\prime} \frac{\delta_{0}}{4}\left(\frac{\pi}{\Psi^{-1}(x)}\right)^{3 / 2} \exp \left(-\frac{\left(\frac{\pi}{2}\right)^{4} \delta_{0}^{2}}{\Psi^{-1}(x)}\right) .
$$


Proof: The proof is an immediate result of Theorem 2 applied to $F_{P_{H}}(x)=F_{P_{r}}\left(\Psi^{-1}(x)\right)$.

Remark 4: If $\Psi(\cdot)$ stands for the linear energy harvesting model, we have $\Psi^{-1}(x)=\frac{x}{\zeta}$, and $\left(\Psi^{-1}(x)\right)^{\prime}=\frac{1}{\zeta}$, where $\zeta \in$ $[0,1]$ is the harvesting efficiency.

In addition, if $\Psi(\cdot)$ stands for the nonlinear sigmoidal energy harvesting model, we have $\Psi(x)=\frac{\frac{M_{e}-M_{e}-M_{e} \Omega}{1+e_{e}\left(x-b_{e}\right)}}{1-\Omega}, \Psi^{-1}(x)=$ $b_{e}-\frac{1}{a_{e}} \log \left(\frac{(1-\Omega)\left(M_{e}-x\right)}{M_{e} \Omega+(1-\Omega) x}\right)$, and $\left(\Psi^{-1}(x)\right)^{\prime}=\frac{M_{e}}{a_{e}\left(M_{e}-x\right)\left(M_{e} \Omega+(1-\Omega) x\right)}$, where $\Omega=\frac{1}{1+e_{e} b_{e}}, M_{e}$ is the maximum harvested energy, $a_{e}$ and $b_{e}$ are experimental parameters [31].

Given that the battery is discretized into $N \times M$ equal levels, the probability of harvesting $0 \leq m \leq N M$ levels of energy is expressed as

$$
p_{m}= \begin{cases}F_{P_{H}}((m+1) \omega)-F_{P_{H}}(m \omega), & \text { if } m=0, \ldots, N M-1, \\ 1-F_{P_{H}}\left(N P_{D}\right), & \text { if } m=N M, \\ 0, & \text { otherwise. }\end{cases}
$$

For the special case of $\alpha_{B}=4$ and the linear energy harvesting model, the derivation of the harvesting probabilities simplifies as in the following corollary:

Corollary 2: For $\alpha_{B}=4$ and the linear energy harvesting model, the harvesting probabilities $p_{m}$ is given by

$p_{m} \underset{\alpha_{B}=4}{=} \begin{cases}\operatorname{erf}\left(\frac{\pi^{2} \delta_{0} \sqrt{\zeta}}{4 \sqrt{m \omega}}\right)-\operatorname{erf}\left(\frac{\pi^{2} \delta_{0} \sqrt{\zeta}}{4 \sqrt{(m+1) \omega}}\right), & \text { if } m=0, \ldots, N M-1, \\ \operatorname{erf}\left(\frac{\pi^{2} \delta_{0} \sqrt{\zeta}}{4 \sqrt{N P_{D}}}\right), & \text { if } m=N M .\end{cases}$

Proof: Follows from Proposition 2 and (13).

Remark 5: The special case of the path loss exponent $\alpha_{B}=4$ is of particular interest because it is a common value for urban outdoor cellular environment. Furthermore, $\alpha_{B}=4$ gives a closed form expression for the PDF of the harvested cellular energy, which simplifies the analysis and numerical computations.

\section{B. Queueing Theory Analysis}

The DTMC in Fig. 2 represents a quasi birth death process (QBD) with geometric (Geo) arrival and phase (PH) type departure, denoted as $\mathrm{Geo} / \mathrm{Ph} / 1$ QBD system [34]. That is, the queue departure process (i.e., transmission success probability) can be represented via two sub-stochastic matrices $\boldsymbol{S}_{N}$ and $\boldsymbol{G}_{N}$ of size $(N M+1) \times(N M+1)$. Let us denote by $\boldsymbol{W}_{M}$ the $M \times(N M+1)$ matrix and $V_{N M}$ the $((N-1) M+1) \times$ $((N-1) M+1)$ matrix defined as

$\boldsymbol{W}_{M}$

$$
=\left[\begin{array}{ccccccc}
p_{0} & & \cdots & p_{(N-1) M} & \cdots & p_{N M-1} & 1-\sum_{m=0}^{N M-1} p_{m} \\
\vdots & \ddots & & & \ddots & \vdots & \vdots \\
0 & \cdots & p_{0} & \ldots & & p_{(N-1) M} & 1-\sum_{m=0}^{(N-1) M} p_{m}
\end{array}\right],
$$

$$
\begin{aligned}
\boldsymbol{V}_{N M} & =p_{c} \boldsymbol{I}_{((N-1) M+1) \times((N-1) M+1)} \\
\text { and } \overline{\boldsymbol{V}}_{N M} & =\left(1-p_{c}\right) \boldsymbol{I}_{((N-1) M+1) \times((N-1) M+1)},
\end{aligned}
$$

respectively. Then, the matrices $\boldsymbol{S}_{N}$ and $\boldsymbol{G}_{N}$ of size $(N M+1) \times$ $(N M+1)$ can be expressed as

$$
\boldsymbol{S}_{N}=\left[\begin{array}{lll} 
& \boldsymbol{W}_{M} & \\
\overline{\boldsymbol{V}}_{N M} & & \mathbf{0}_{((N-1) M+1) \times M}
\end{array}\right],
$$

$$
\boldsymbol{G}_{N}=\left[\begin{array}{lll} 
& \mathbf{0}_{M \times(N M+1)} & \\
\boldsymbol{V}_{N M} & & \mathbf{0}_{((N-1) M+1) \times M}
\end{array}\right] .
$$

The matrix $\boldsymbol{S}_{N}$ is an $(N M+1) \times(N M+1)$ sub-stochastic matrix that models the energy harvesting/depletion process until one successful packet departure. Transmission attempts take place when the battery has at least $M$ levels of energy. With each transmission, the battery is depleted with $M$ levels of energy. The initialization vector for the $\mathrm{PH}$ type process is given by the matrix $\boldsymbol{G}_{N}$. When the IoT device has an empty buffer, it harvests energy up to full battery and waits for a packet arrival. Hence, the energy harvesting matrix at level-0 is a stochastic matrix given by the matrix $S_{0, N}$ of size $(N M+1) \times(N M+1)$ is defined as

$$
\boldsymbol{S}_{0, N}=\left[\begin{array}{ccccc}
p_{0} & p_{1} & \ldots & p_{N M-1} & 1-\sum_{i=0}^{N M-1} p_{i} \\
0 & p_{0} & \ldots & p_{N M-2} & 1-\sum_{i=0}^{N M-2} p_{i} \\
\vdots & \ddots & \ddots & \vdots & \vdots \\
0 & \ldots & 0 & p_{0} & 1-p_{0} \\
0 & \ldots & \ldots & 0 & 1
\end{array}\right] .
$$

Utilizing the matrices described above, the transition matrix of the Geo/PH/1 system shown in Fig. 2 is given by

$$
\boldsymbol{P}=\left[\begin{array}{ccccc}
\boldsymbol{B} & \boldsymbol{C} & & & \\
\boldsymbol{A}_{2} & \boldsymbol{A}_{1} & \boldsymbol{A}_{0} & & \\
& \boldsymbol{A}_{2} & \boldsymbol{A}_{1} & \boldsymbol{A}_{0} & \\
& & \ddots & \ddots & \ddots
\end{array}\right],
$$

where $\boldsymbol{B}=\bar{a} \boldsymbol{S}_{0, N}$ and $\boldsymbol{C}=a \boldsymbol{S}_{0, N}$ are sub-stochastic matrices that contain the transition probabilities within the idle state and from the idle-to-level 1 (i.e., first packet arrival), respectively. The sub-stochastic matrices $\boldsymbol{A}_{0}=\bar{a} \boldsymbol{G}_{N}, \boldsymbol{A}_{1}=\bar{a} \boldsymbol{S}_{N}+a \boldsymbol{G}_{N}$, and $\boldsymbol{A}_{2}=a \boldsymbol{S}_{N}$ contain the joint buffer and battery states transitions probabilities when going up one level, staying within the same level, and going down one level, respectively.

Let $\boldsymbol{x}=\left[\begin{array}{llll}x_{0} & \boldsymbol{x}_{1} & \boldsymbol{x}_{2} & \ldots\end{array}\right]$, where $\boldsymbol{x}_{i}=\left[\begin{array}{lll}x_{i, 0} & \ldots & x_{i, N M}\end{array}\right]$ denotes the probability of having $i$ packets in the data buffer and $x_{i, m}$ is the probability of having $i$ packets and $m$ energy levels. The steady state vector $\boldsymbol{x}$ is obtained by solving

$$
\boldsymbol{x}=\boldsymbol{x} \boldsymbol{P} \quad \text { and } \quad \boldsymbol{x} \mathbf{1}=1,
$$

where $\mathbf{1}$ is an infinite column vector of ones. A unique solution for the system of equations in (21) exists if the DTMC in Fig. 2 is stable. The stability conditions is characterized as follows:

Lemma 1: A data buffer of an IoT device is stable if

$$
\pi_{N} A_{2} e>\pi_{N} A_{0} e
$$

where $\boldsymbol{\pi}_{N}$ is defined as

$$
\boldsymbol{\pi}_{N}=\left(\boldsymbol{S}_{N}+\boldsymbol{G}_{N}-\boldsymbol{I}+\boldsymbol{e} \boldsymbol{e}^{T}\right)^{-1} \boldsymbol{e},
$$

$\boldsymbol{e}$ is the column vector of ones of size $(N M+1)$, and $\boldsymbol{I}$ is the identity matrix. Otherwise, the data buffer is unstable and the packet delay is infinite.

Proof: According to [34], a $\mathrm{Geo} / \mathrm{PH} / 1$ QBD system is stable if $\boldsymbol{\pi}_{N} \boldsymbol{A}_{2} \boldsymbol{e}>\boldsymbol{\pi}_{N} \boldsymbol{A}_{0} \boldsymbol{e}$, where $\boldsymbol{\pi}_{N}$ is solution to

$$
\boldsymbol{\pi}_{N}=\boldsymbol{\pi}_{N} \boldsymbol{A} \text { and } \boldsymbol{\pi}_{N} \boldsymbol{e}=1
$$

where $\boldsymbol{A}=\boldsymbol{A}_{0}+\boldsymbol{A}_{1}+\boldsymbol{A}_{2}$. Substituting for $\boldsymbol{A}_{0}=a \boldsymbol{S}_{N}, \boldsymbol{A}_{1}=$ $\bar{a} \boldsymbol{S}_{N}+a \boldsymbol{G}_{N}$, and $\boldsymbol{A}_{2}=\bar{a} \boldsymbol{G}_{N}$ and using [35, Lemma 1] to solve (24), the lemma is proved.

The subsequent analysis depends on the output of the stability condition in (22) as shown in the sequel. 
1) Stable system: If the condition in (22) is satisfied, the solution for the DTMC in (21) is given as:

Theorem 3: The joint steady state probabilities for the number of packets in the data buffer and energy levels in the battery of a typical IoT device is given by

$$
\boldsymbol{x}_{i}= \begin{cases}a \boldsymbol{x}_{0} \boldsymbol{S}_{0, N}\left(\boldsymbol{I}-a \boldsymbol{G}_{N}-\bar{a} \boldsymbol{S}_{N}-\boldsymbol{R} \bar{a} \boldsymbol{G}_{N}\right)^{-1} & \\ \bar{a} \boldsymbol{G}_{N}\left(\boldsymbol{I}-\bar{a} \boldsymbol{S}_{0, N}\right)^{-1}, & \text { if } i=0, \\ a \boldsymbol{x}_{0} \boldsymbol{S}_{0, N}\left(\boldsymbol{I}-a \boldsymbol{G}_{N}-\bar{a} \boldsymbol{S}_{N}-\boldsymbol{R} \bar{a} \boldsymbol{G}_{N}\right)^{-1}, & \text { if } i=1, \\ \boldsymbol{x}_{1} \boldsymbol{R}^{i-1}, & \text { if } i>1,\end{cases}
$$

satisfying $\boldsymbol{x}_{0} \boldsymbol{e}+a \boldsymbol{x}_{0} \boldsymbol{S}_{0, N}\left(\boldsymbol{I}-a \boldsymbol{G}_{N}-\bar{a} \boldsymbol{S}_{N}-\boldsymbol{R} \bar{a} \boldsymbol{G}_{N}\right)^{-1}(\boldsymbol{I}-\boldsymbol{R})^{-1} \boldsymbol{e}=$ 1, with with $\boldsymbol{R}$ is the minimum of the matrix quadratic equation $\boldsymbol{R}=\boldsymbol{A}_{0}+\boldsymbol{R} \boldsymbol{A}_{1}+\boldsymbol{R}^{2} \boldsymbol{A}_{2}$.

Proof: Following [34], $\boldsymbol{x}_{0}$ and $\boldsymbol{x}_{1}$ are the solutions to:

$$
\left(\begin{array}{ll}
x_{0} & x_{1}
\end{array}\right)=\left(\begin{array}{ll}
x_{0} & x_{1}
\end{array}\right)\left(\begin{array}{cc}
B & C \\
A_{2} & A_{1}+R A_{2}
\end{array}\right),
$$

where $\boldsymbol{R}$ is the minimal non-negative solution of the matrix quadratic equation $\boldsymbol{R}=\boldsymbol{A}_{0}+\boldsymbol{R} \boldsymbol{A}_{1}+\boldsymbol{R}^{2} \boldsymbol{A}_{2}$.

If the inequality in (22) is satisfied, the network is said to be stable (i.e., packets are delivered in finite time) and $\boldsymbol{R}$ is known to have a spectral radius less than one. Hence, following [34] and solving (26), $\boldsymbol{x}_{0}$ is obtained as:

$$
\boldsymbol{x}_{0}=a \boldsymbol{x}_{0} \boldsymbol{S}_{0, N}\left(\boldsymbol{I}-a \boldsymbol{G}_{N}-\bar{a} \boldsymbol{S}_{N}-\boldsymbol{R} \bar{a} \boldsymbol{G}_{N}\right)^{-1} \bar{a} \boldsymbol{G}_{N}\left(\boldsymbol{I}-\bar{a} \boldsymbol{S}_{0, N}\right)^{-1},
$$

with the normalization condition:

$\boldsymbol{x}_{0} \boldsymbol{e}+a \boldsymbol{x}_{0} \boldsymbol{S}_{0, N}\left(\boldsymbol{I}-a \boldsymbol{G}_{N}-\bar{a} \boldsymbol{S}_{N}-\boldsymbol{R} \bar{a} \boldsymbol{G}_{N}\right)^{-1}(\boldsymbol{I}-\boldsymbol{R})^{-1} \boldsymbol{e}=1 . \boldsymbol{x}_{1}$ is deduced from (26) as

$$
\boldsymbol{x}_{1}=a \boldsymbol{x}_{0} \boldsymbol{S}_{0, N}\left(\boldsymbol{I}-a \boldsymbol{G}_{N}-\bar{a} \boldsymbol{S}_{N}-\boldsymbol{R} \bar{a} \boldsymbol{G}_{N}\right)^{-1} .
$$

For $i \geq 2, \boldsymbol{x}_{i}$ is given by $\boldsymbol{x}_{i}=\boldsymbol{x}_{1} \boldsymbol{R}^{i-1}$.

Since $\boldsymbol{S}$ and $\boldsymbol{G}$ are not of rank one, the matrix $\boldsymbol{R}$ has no closedform expression and is obtained by solving Algorithm 1 .

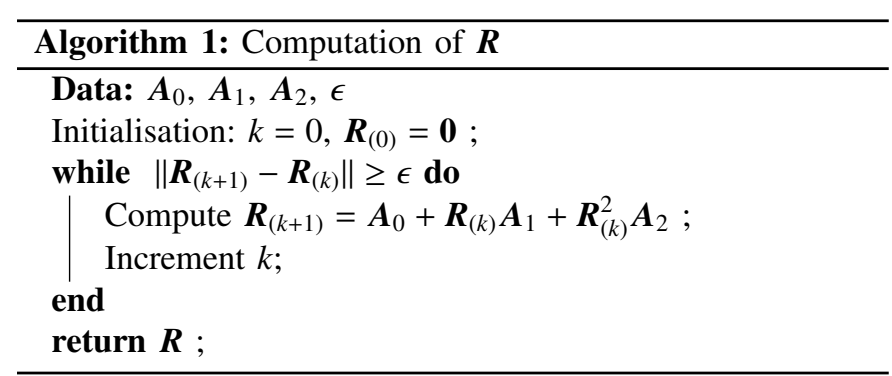

Let $\boldsymbol{\Phi}_{p}=\left[\Phi_{p, 0}, \Phi_{p, 1}, \ldots, \Phi_{p, N M}\right]$ where $\Phi_{p, m}$ is the marginal probability of having $m$ units of energy levels in the battery for a non-empty data buffers. If the system is stable, the battery level marginal distribution is characterized in the following corollary:

Corollary 3: The marginal distribution of the phases with nonempty data buffers is given by

$$
\boldsymbol{\Phi}_{p}=\sum_{i \geq 1} \boldsymbol{x}_{i}=\boldsymbol{x}_{1}(\boldsymbol{I}-\boldsymbol{R})^{-1} \text {. }
$$

Proof: Let $\boldsymbol{\Phi}=\left[\Phi_{0}, \Phi_{1}, \ldots, \Phi_{M N}\right]$ where $\Phi_{m}$ be the marginal probability of having $m$ units of energy levels in the battery. The marginal distribution of the phases is given by $\boldsymbol{\Phi}=\sum_{i \geq 0} \boldsymbol{x}_{i}=\boldsymbol{x}_{0}+x_{1} \sum_{i \geq 1} \boldsymbol{R}^{i-1}=\boldsymbol{x}_{0}+\boldsymbol{x}_{1}(\boldsymbol{I}-\boldsymbol{R})^{-1}$. Since $\boldsymbol{\Phi}=\boldsymbol{x}_{0}+\boldsymbol{\Phi}_{p}$, we deduce (29).
Since an IoT device transmits when it has non-empty buffer and more than $M$ levels of energy in his battery, hence, the activity probability $d$ is

$$
d=\sum_{n=M}^{N M} \Phi_{p, n} .
$$

2) Unstable system: If the condition in (22) is not satisfied, then the DTMC is unstable and Theorem 1 and Corollary 3 are no more applicable. This is because the departure rate is less than the arrival rate [34], and hence, the data buffer accumulates infinite number of packets and the probability of having empty buffer is nullified. Although unstable buffers are never empty, an IoT device does not transmit unless it has full battery. Hence, only the marginal phase distribution for the battery is considered. This fact translates into setting $\boldsymbol{x}_{0}=0$, which leads to $\boldsymbol{\Phi}_{p}=\left(\boldsymbol{S}_{N}+\boldsymbol{G}_{N}-\boldsymbol{I}+\boldsymbol{e} \boldsymbol{e}^{T}\right)^{-1} \boldsymbol{e}$. The transmission probability for unstable system is also given by (30).

\section{Iterative Solution}

As mentioned earlier, there is an interdependence between the microscopic device behavior and the macroscopic mutual interaction between IoT devices. Particularly, the aggregate interference seen by the typical IoT device is function of the activity of other IoT devices. Hence, the transmission success probability in (2) requires the probability that an IoT device is active, given by (30). Meanwhile, the queueing theory analysis for computing the activity probability in (30) requires the transmission success probability as shown in (15) and (16). Such interdependence is solved via the iterative solution in Algorithm 2, which converges to a unique solution by virtue of fixed point theorem [36, Appendix B].

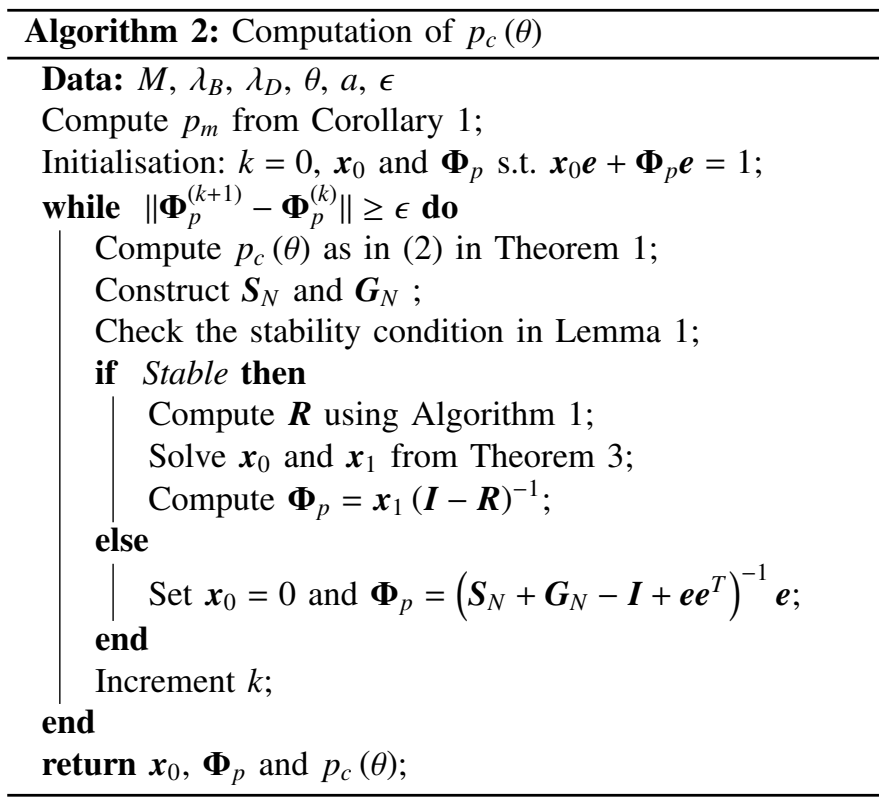

\section{Performance Metrics}

After solving the iterative solution, we study some key performance indicators of IoT networks. For stable networks, 
we can characterize the average queue length $\mathbb{E}\left[Q_{L}\right]$ and the average queueing delay $\mathbb{E}\left[W_{q}\right]$, where $Q_{L}$ is the instantaneous queue length and $W_{q}$ is the queue delay for a randomly selected packet. The average queue length is given by

$$
\begin{aligned}
\mathbb{E}\left[Q_{L}\right] & =\sum_{i=2}^{\infty}(i-1) \mathbb{P}\left[Q_{L}=i\right]=\sum_{i=2}^{\infty}(i-1) \sum_{m=0}^{M} \boldsymbol{x}_{i, m} \\
& =\sum_{i=2}^{\infty}(i-1) \boldsymbol{x}_{i} \boldsymbol{e}=\boldsymbol{x}_{1} \boldsymbol{R}(\boldsymbol{I}-\boldsymbol{R})^{-2} \boldsymbol{e} .
\end{aligned}
$$

Similarly, the average number of packets handled by an IoT device including the one in service is given by

$$
\mathbb{E}[X]=\sum_{i=1}^{\infty} i \boldsymbol{x}_{i} \boldsymbol{e}=\boldsymbol{x}_{1} \sum_{i=1}^{\infty} i \boldsymbol{R}^{i-1} \boldsymbol{e}=\boldsymbol{x}_{1}(\boldsymbol{I}-\boldsymbol{R})^{-2} \boldsymbol{e} .
$$

Applying the Little's law for stable queues, the average waiting time is the ratio between the average number of packets in the system over the packet arrival rate $a$

$$
\mathbb{E}\left[W_{q}\right]=\frac{\mathbb{E}[X]}{a}=\frac{\boldsymbol{x}_{1}(\boldsymbol{I}-\boldsymbol{R})^{-2} \boldsymbol{e}}{a} .
$$

The temporal (i.e., across different packets) distribution of the delay can be obtained as

$$
\mathbb{P}\left[W_{q}=0\right]=\boldsymbol{x}_{0} \boldsymbol{e}, \quad \text { and } \quad \mathbb{P}\left[W_{q}=j\right]=\sum_{k=1}^{j} \boldsymbol{x}_{k} \boldsymbol{B}_{j}^{(k)} \boldsymbol{e},
$$

where $\boldsymbol{B}_{j}^{(k)}$ represents the probability of having $k$ packets in the queue and being serviced in $j$ times

$$
\boldsymbol{B}_{j}^{(k)}= \begin{cases}\boldsymbol{S}^{j-1} \boldsymbol{G}_{N}, & \text { if } k=1, \\ \boldsymbol{S B}_{j-1}^{(k)}+\boldsymbol{G}_{N} \boldsymbol{B}_{j-1}^{(k-1)}, & \text { if } 1<k<j, \\ \boldsymbol{G}_{N}^{k}, & \text { if } k=j,\end{cases}
$$

with $1 \leq k \leq j$.

\section{Numerical Results}

In this section, we first validate the proposed spatiotemporal mathematical framework against Monte Carlo simulations. Then, we discuss the self-sustainability of the depicted IoT network. Unless otherwise stated, the network parameters are selected as follows: the transmit power of all BSs is $P_{B}=1$ $\mathrm{W}$, the power control parameter is $\rho_{D}=-20 \mathrm{dBm}$, the IoT receiver separation distance is $r_{0}=2$ meters, the path loss exponents are $\alpha_{B}=4$ and $\alpha_{D}=3$, the conversion efficiency is $\zeta=0.6$, and the number of battery levels is $M=4$. The number of Monte Carlo simulations is 5000 .

\section{A. Validation of Analytical Expressions}

The Monte Carlo simulations are performed over a network area of $100 \times 100 \mathrm{~m}^{2}$ with a wrapped around boundaries. Two independent realizations of a PPP and a PBP, with intensities $\lambda_{B}=10^{-2} \mathrm{BSs} / \mathrm{m}^{2}$ and $\lambda_{D}=510^{-2} \mathrm{IoTs} / \mathrm{m}^{2}$, are used respectively for the cellular and IoT networks. The realizations of the PPP and PBP are kept fixed, while fading and devices/BSs activities change over time. Each Monte Carlo simulation run is considered as a time slot where independent channel gains are instantiated, packets are generated, energy is harvested by some IoT devices and packets are transmitted by other IoT devices (i.e., those with non-empty buffers and full batteries). The time evolves and the transmission success probabilities and steady state statistics are traced until steady state, where the statistics are collected. This process is repeated several times with different realizations of the PPP and PBP for the sake of spatial averaging.

In Fig. 3(a), we show the harvesting probability mass function $p_{m}$, with $0 \leq m \leq M N$, for $b=0.1, M=4$, $N=4$, and $N_{c}=5$. The analytic expressions and Monte Carlo simulations are both plotted. The agreement between the analytic expressions and the Monte Carlo simulations in Fig. 3(a) validates our analysis for the harvesting probability.

In Figs. 3(b), 3(c), 3(d), we plot the analytical expression (i.e., Algorithm 2) and Monte Carlo simulations for the packet transmission success probability $p_{c}(\theta)$ versus the detection threshold $\theta$ in $\mathrm{dB}$ for $b=0.1, M=8, N=1$, and $N_{c}=1$. In Fig. 3(b), the traffic arrival probability $a$ is either $a=0.1$ or $a=0.2$, with $b=1$. In Fig. 3(c), the EH model is either linear $(\zeta=0.6)$ or the nonlinear sigmoidal model $\left(a_{e}=1500\right.$, $b_{e}=0.0022, M_{e}=24 \mathrm{~mW}$ ) [31], with $a=b=0.1$. In Fig. $3(\mathrm{~d})$, the receivers are uniformly distributed in a disc of radius $R_{c}$ around the transmitters, where the PDF of the distance $r_{0}$ is $f_{r_{d}}(r)=\frac{2 r}{R_{c}^{2}}$ with $0 \leq r \leq R_{c}$, with $a=b=0.1$ and $R_{c}=2$ m. Unless specified in all Figs. 3(b), 3(c), 3(d), the EH model is linear and the distance $r_{0}$ is fixed.

First of all, the agreement between the analytic expressions and the Monte Carlo simulations is verified in Figs. 3(b) and 3(d) which validates our analysis. Moreover, as expected, all figures 3(b), 3(c), 3(d) confirm that as the detection threshold $\theta$ increases, the packet transmission success probability decreases. This leads to more packet accumulation in the IoT devices' buffers and longer activity per IoT device. Consequently, the mutual interference between IoT devices increases. These figures confirm the intuition that there is a number of critical points at which the system becomes unstable, which occurs when the departure probability is less than the arrival rate. In Fig. 3(b), the critical thresholds for $a=0.2$ and $a=0.1$ are $\theta=5 \mathrm{~dB}$ and $\theta=10 \mathrm{~dB}$, respectively. Indeed the critical threshold for $a=0.1$ is larger than that of $a=0.2$, which is confirmed and quantified in the figure. A network with $a=0.1$ has less active devices than a network with $a=0.2$, and hence, $a=0.1$ implies less mutual interference and higher departure probability. The figure also shows that for unstable network operation, the transmission success probability becomes independent of $a$. That is, at unstable network operation the probability for being idle is zero and all devices are active irrespective of the actual value of $a$.

\section{B. Unsaturated and Saturated Buffer/Battery}

In addition, Fig. 3(c) compares the four scenarios of saturated/unsaturated data buffers and batteries. Fig. 3(c) compares also the use of linear and nonlinear EH models. Fig. 3(d) exhibits the use of the random uniform distribution of $r_{0}$. In general, the figures 3(c), 3(d) show that the highest transmission success probability is achieved when both the buffer and battery are assumed to be unsaturated. That is, ignoring the randomness in the packets and/or energy generation 


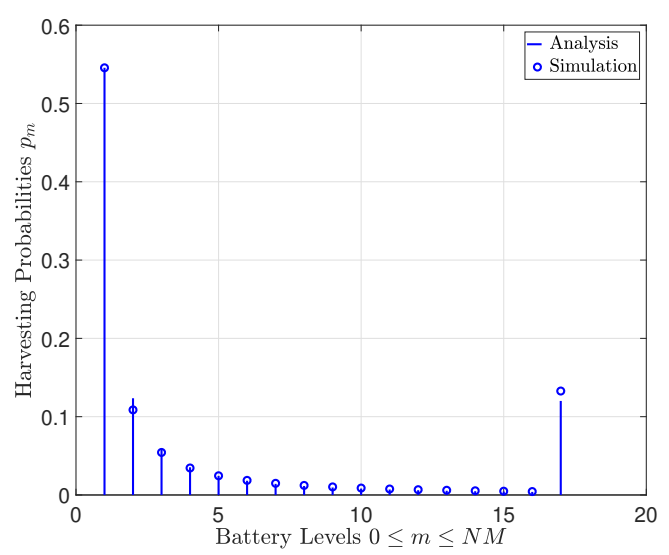

(a) $p_{m}$ with $b=0.1, M=4, N=4$, and $N_{c}=5$.

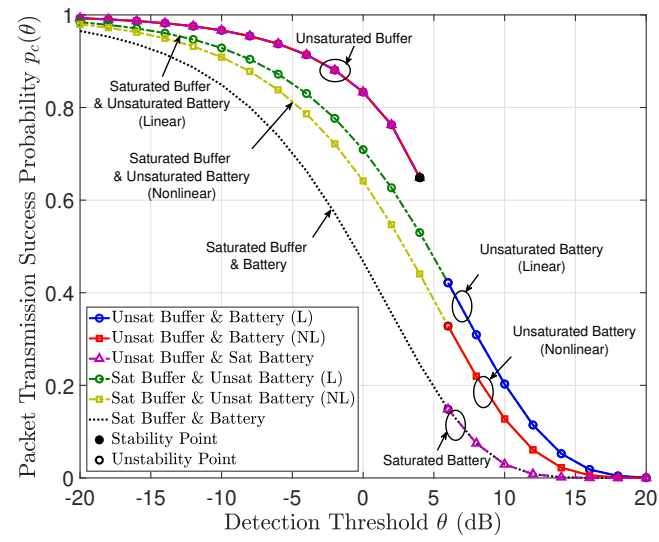

(c) $p_{c}(\theta)$ with nonlinear vs linear EH model with $a=b=$ 0.1 , and $N_{c}=N=1$.

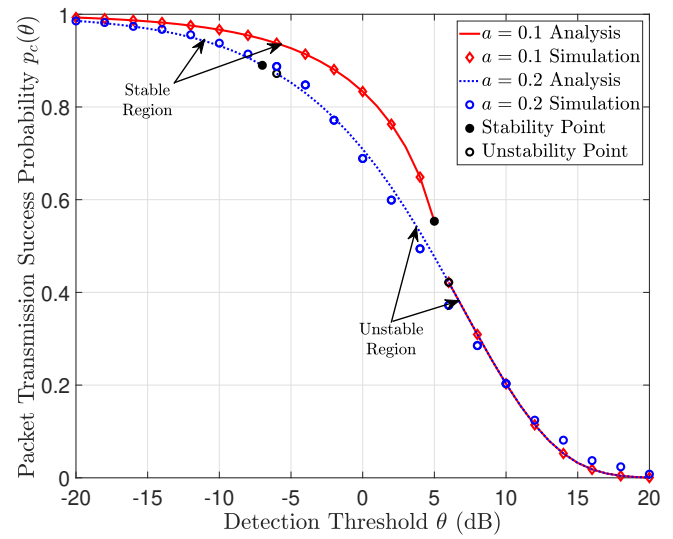

(b) $p_{c}(\theta)$ with different packet arrival probabilities with $b=$ 1 , and $N_{c}=N=1$.

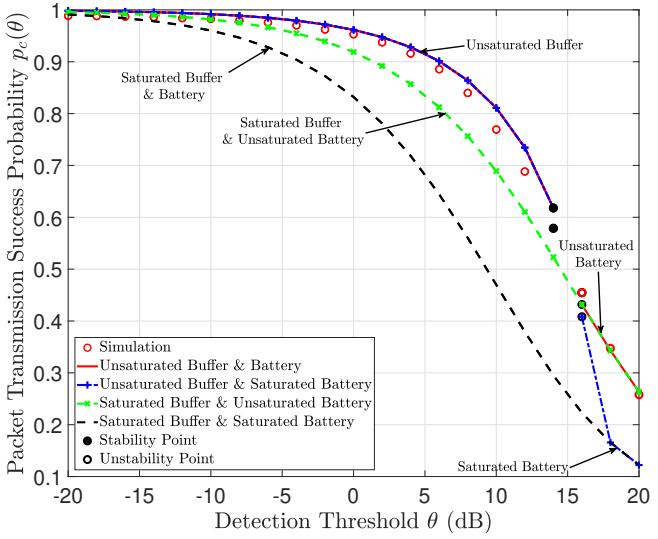

(d) $p_{c}(\theta)$ with random IoT Tx-Rx distances with $a=b=$ 0.1 , and $N_{c}=N=1$.

Figure 3. Harvesting probability mass function $p_{m}$ and packet transmission success probability $p_{c}(\theta)$ versus the detection threshold $\theta$ in $\mathrm{dB}$ with $M=8$.

will lead to overestimated mutual interference and pessimistic assessment for the transmission success probability. More specifically, Fig. 3(c) shows that as long as the network is stable, the curves with unsaturated buffers (i.e., red, blue and purple) coincide. Same remark for Fig. 3(d) where red and blue curves coincide. This is because energy harvesting occurs at a faster rate than packet generation, and hence, the spectrum access and mutual interference are dominated by the packet generation process. That is why, in Fig. 3(c), the linear and nonlinear models have no impact on the performance with unsaturated buffer and stable network (red and blue curves coincide).

However, when the network is unstable (i.e., there are always packets in the buffers), the energy harvesting becomes the dominant factor for spectrum access and mutual interference. Hence, the saturated battery assumption (i.e., purple curve in Fig. 3(c), and blue curve in 3(d)) leads to pessimistic assessment for the transmission success probability when compared to the unsaturated battery scenario (i.e., blue and red curves in Fig. 3(c), and red curve in 3(d)). In addition, the linear model leads to higher transmission success probability compared to the nonlinear model for unstable networks with saturated buffers. Fig. 3(c) also shows that the saturated buffer assumption (i.e., the light green, dark green and black curves) are always underestimating the transmission success probability when compared to the unsaturated buffers stable regimes. The underestimation is more severe for saturated battery (i.e., black curve) as all devices always mutually interfere together. Same observation applies to 3(d). Furthermore, there is no notion of instability in the saturated buffer scenario. In addition, assuming random distance distribution exhibits higher transmission success probability when comparing Figs. $3(\mathrm{c})$, and $3(\mathrm{~d})$, and this confirms that fixing the distance is a pessimistic assessment of the network and helps only to simplify the analysis and is not overestimating the network performance. Last but not least, the unsaturated buffer curves coincide with their corresponding saturated buffer curves when the network is unstable, which shows that the unsaturated buffer assumption intrinsically accounts for the case of high traffic where all devices would always have packets in their buffers. Hence, the unsaturated buffer and energy assumption are the most general and practical assumptions which capture the saturated scenarios as special cases.

\section{Self-Sustainability Regions}

In Figs. 4(a), 4(b), and 4(c), we show the self-sustainability region versus the spatiotemporal IoT traffic density $\left(a, \lambda_{D}\right)$, with $b=0.1, N_{c}=8$, and $\theta=10 \mathrm{~dB}$. In Fig. 4(a), we 


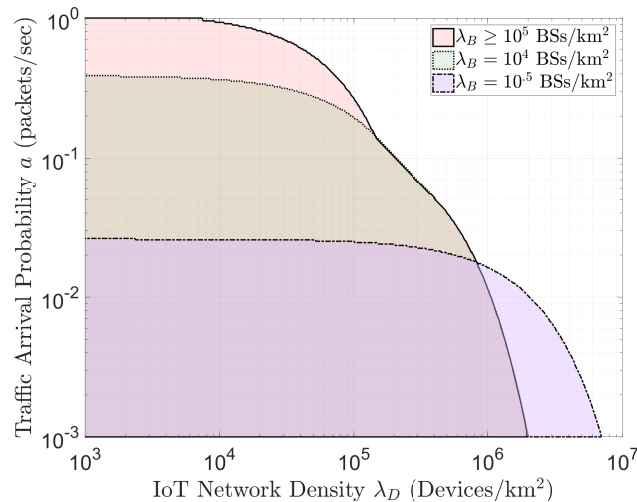

(a) Different cellular network densities $\lambda_{B}$ in $\mathrm{BSs} / \mathrm{km}^{2}$, with $M=8$ and $N=4$.

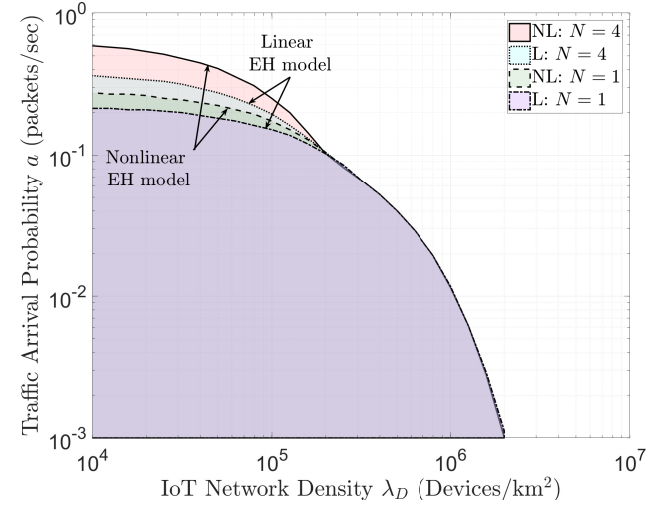

(b) Nonlinear vs linear EH model with $\lambda_{B}=10^{4} \mathrm{BSs} / \mathrm{km}^{2}$, and $M=8$.

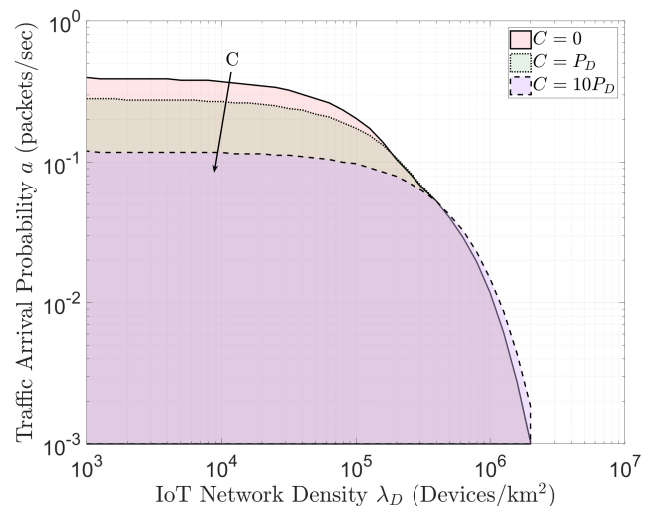

(c) Other sources of power consumption $C$, with $\lambda_{B}=10^{4}$ $\mathrm{BSs} / \mathrm{km}^{2}$, and $M=1$ and $N=4$.

Figure 4. Stability Pareto regions $\left(a, \lambda_{D}\right)$ between the packet arrival probability $a$ in packets/sec versus the IoT network density $\lambda_{D}$ in IoTs/km ${ }^{2}$ with $b=0.1$, $N_{c}=8$, and $\theta=10 \mathrm{~dB}$.

have different cellular BSs intensities $\lambda_{B}$ in $\mathrm{BSs} / \mathrm{km}^{2}$, with $M=8$, and $N=4$. In Fig. 4(b), we consider either the linear EH model $(\zeta=0.6)$ or the nonlinear sigmoidal $\mathrm{EH}$ model $\left(a_{e}=1500, b_{e}=0.0022, M_{e}=24 \mathrm{~mW}\right)$ [31], with $\lambda_{B}=10^{4} \mathrm{BSs} / \mathrm{km}^{2}, M=8$, and $N=1$ or $N=4$. In Fig. 4(c), we have different values for the other sources of power consumption (not only data transmission) (please refer to footnote 7), with $\lambda_{B}=10^{4} \mathrm{BSs} / \mathrm{km}^{2}, M=1$, and $N=4$. The shaded regions in Figs. 4(a), 4(b), and 4(c) (as well as the following figures) determine the pairs $\left(a, \lambda_{D}\right)$ that lead to stable network operation. That is, the harvesting rate is quick enough and the mutual interference is tolerable to maintain packet departure rates that are greater than the packet arrival rates. Consequently, all generated packets get delivered to the intended receivers in finite time. Operating beyond the shaded regions means that the packet departure rates is less than the packet arrival rate, which leads to infinite packet accumulation in the IoT devices' buffers and infinite average delay. Instability can occur due to i) energy scarcity (i.e., slow harvesting rates), ii) overwhelming interference (i.e., low transmission success probability), or iii) both reasons in i) and ii).

It can be deduced from Fig. 4(a) that for low and medium device densities (i.e., up to $10^{4}$ device $/ \mathrm{km}^{2}$ ), energy scarcity is the prominent factor for instability. Hence, increasing the
BSs density extend the sustainability region, for this range of device intensities, as it improves the harvesting rates to cope with the higher traffic arrivals. This is confirmed in 4(b) for $\lambda_{B}=10^{4} \mathrm{BSs} / \mathrm{km}^{2}$ where varying the EH model (linear or nonlinear) leads only to extend the sustainability region for low and medium device densities. Also, increasing the power consumed from other sources (not only data consumption) reduces the sustainability region only for low and medium device densities in Fig. 4(c). In contrast, at high devices density (beyond $10^{5}$ device $/ \mathrm{km}^{2}$ ), the interference becomes the dominant obstacle for stability, where several retransmissions are required to successfully deliver each packet. Hence, increasing the BS intensity as in Fig. 4(a), or changing the EH model as in Fig. 4(b) does not extend the sustainability region for higher IoT device intensity. Interestingly, Fig. 4(a) shows that lower BS intensity can accommodate higher IoT devices intensity (e.g., for $a=0.001$ ). Such counterintuitive behavior is because longer harvesting time defers IoT device transmissions and suppresses the aggregate interference. Hence, energy harvesting acts as a distributed spectrum access coordination scheme, which improves transmission success probability and extends the sustainability region for higher IoT device density. This highlights the importance of distributed interference management for self-sustainable massive IoT networks.

In Figs. 5(a) and 5(b), we plot the self-sustainability 


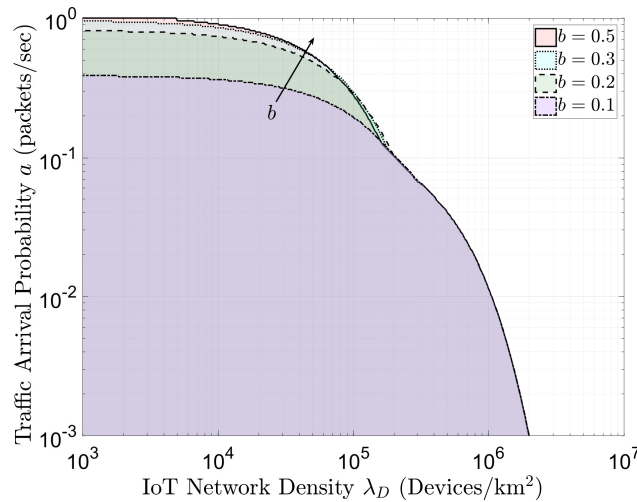

(a) Different cellular base stations activities with $N=4$, $N_{c}=8$.

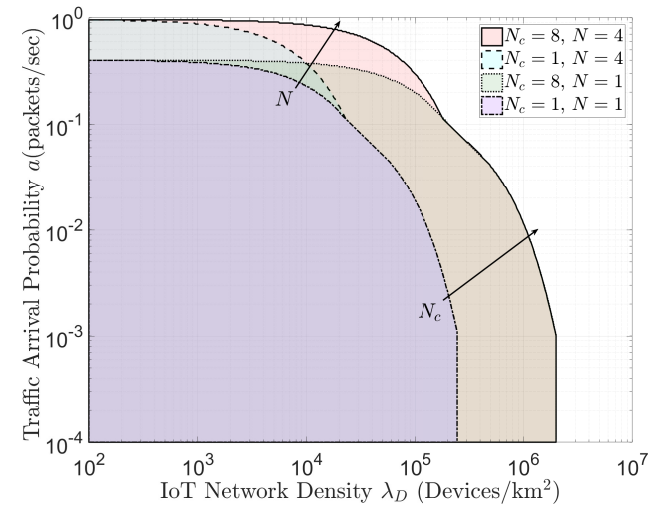

(b) Different number of transmitted packets $N$ and number of channels $N_{c}$, with $b=0.3$.

Figure 5. Stability Pareto regions $\left(a, \lambda_{D}\right)$ between the packet arrival probability $a$ in packets $/ \mathrm{sec}$ versus the IoT network density $\lambda_{D}$ in IoTs $/ \mathrm{km}^{2}$ with $\lambda_{B}=10^{4}$ $\mathrm{BSs} / \mathrm{km}^{2}, \theta=10 \mathrm{~dB}$, and $M=8$.

region versus the spatiotemporal IoT traffic density $\left(a, \lambda_{D}\right)$ with $M=8, \lambda_{B}=10^{4} \mathrm{BSs} / \mathrm{km}^{2}$, and $\theta=10 \mathrm{~dB}$. In Fig. 5(a), we have different cellular base stations activities $b$, with $N=4$, and $N_{c}=8$. In Fig. 5(b), we have different battery capacities (i.e., captured by $N$, the number of transmission attempts a full battery can provide) and different number of channels $N_{c}$, with $b=0.3$. For low and medium device densities (i.e., up to $10^{4}$ device $/ \mathrm{km}^{2}$ ), increasing the BSs activity $b$ or increasing the number $N$ of transmission attempts a full battery can make, extend the sustainability region by increasing the harvesting rates in Figs. 5(a) and 5(b), respectively. As $b$ is greater than 0.5 , no much improvement in the sustainability region can be observed which shows the limitation due to energy scarcity. For higher devices density, no improvement can be observed out of increasing $b$ or $N$ since this region is limited by the interference and the network congestion. To extend the self-sustainability region to higher devices density, it is required to have more channels. Increasing the number of channels that the IoT devices can access diversifies interference, enhances the departure probability, and hence, enables denser IoT networks to be self-sustainable.

\section{Key Performance Indicators}

In Fig. 6, we plot the average queue length versus the detection threshold $\theta$ in $\mathrm{dB}$ for $M=8$, and $\lambda_{B}=\lambda_{D}=-20 \mathrm{~dB}$ with different packet arrival probability $a$, different BS activities, different number of channels, and different number of transmitted packets. The figure shows that the average queue length increases as the detection threshold $\theta$ increases. It is also shown that the average queue length decreases when having lower data arrival rate $a$, more BSs activity $b$, more access channels $N_{c}$, and more number of packets $N$ to transmit. The figure also quantifies the buffer size required for different network parameters, which is crucial for the design of IoT devices.

In Fig. 7, we show the average queueing delay versus the detection threshold $\theta$ in $\mathrm{dB}$ for $M=8$, and $\lambda_{B}=\lambda_{D}=$ $-20 \mathrm{~dB}$ with different packet arrival probability $a$, different BS activities, different number of channels, and different number of transmitted packets. The figure shows that the average queueing delay increases as we increase the detection threshold $\theta$. Moreover, the average queueing delay decreases as $a$ decreases, $b$ increases, $N_{c}$ increases, and $N_{p}$ increases. The figure also quantifies the queueing delay for different network parameters, which is crucial for delay tolerant IoT applications.

\section{Conclusion}

Using stochastic geometry and queueing theory, this paper develops a spatiotemporal mathematical model for selfsustainable IoT networks that recycles the RF-energy of downlink cellular network. Particularly, a two-dimensional discrete time Markov chain (DTMC) is used to track the time evolution of the data buffer and the battery of each IoT device. The IoT network is then modeled as a network of spatially interacting DTMCs due to the mutual interference between IoT devices. To this end, joint probabilities of the number of packets in the buffers and the energy levels in the batteries are obtained. The developed model is then used to characterize and quantify the network self-sustainability and key performance indicators, in terms of spatiotemporal IoT traffic intensity and cellular network density. The results identify the scenarios where the IoT network fails to be self-sustainable due to energy scarcity, overwhelming interference, or both. The results quantify the required buffer size and packet delay, which are crucial for the design of IoT devices and time critical IoT applications.

\section{APPENDix A \\ Laplace Transform of Interfering IoT Devices}

Due to the independence between the channels and the moment generating function (MGF) of the exponential distribution, the LT of the aggregate interference in the IoT network is given by

$$
\begin{aligned}
\mathcal{L}_{D}(s) & \stackrel{(a)}{=} \mathbb{E}_{y_{j}}\left[\prod_{y_{j} \in \Phi_{D} \backslash y_{0}} \mathbb{E}_{g_{j}}\left[\exp \left(-s P_{D} g_{j}\left\|y_{j}\right\|^{-\alpha_{D}}\right)\right]\right] \\
\stackrel{(b)}{=} & \mathbb{E}_{y_{j}}\left[\prod_{y_{j} \in \Phi_{D} \backslash y_{0}} \frac{1}{1+s P_{D}\left\|y_{j}\right\|^{-\alpha_{D}}}\right]
\end{aligned}
$$




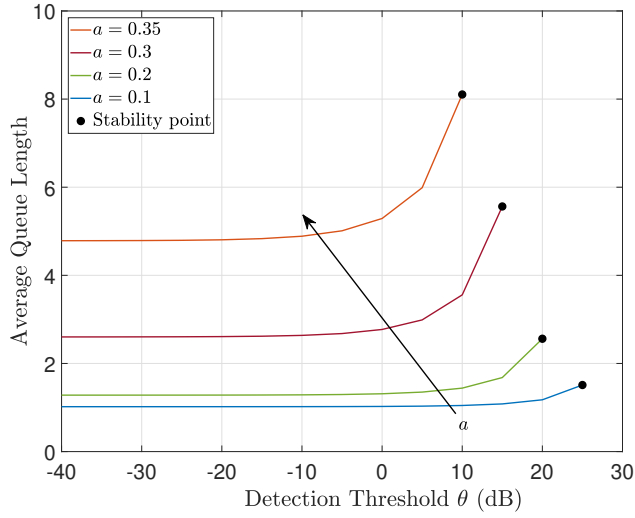

(a) $b=0.1, N_{c}=8, N=4$

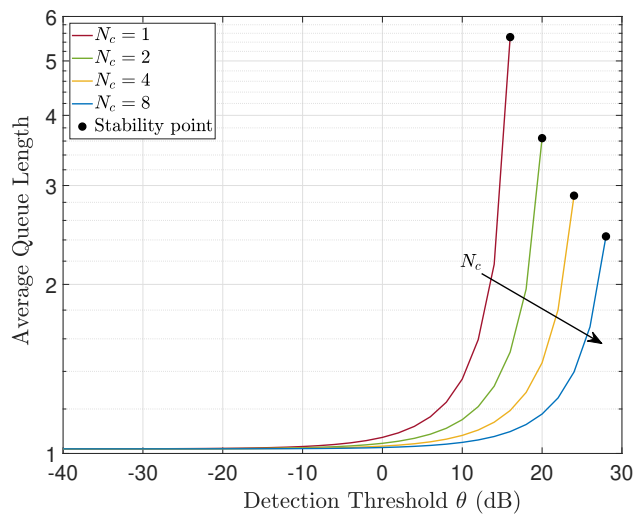

(c) $a=b=0.1, N=4$

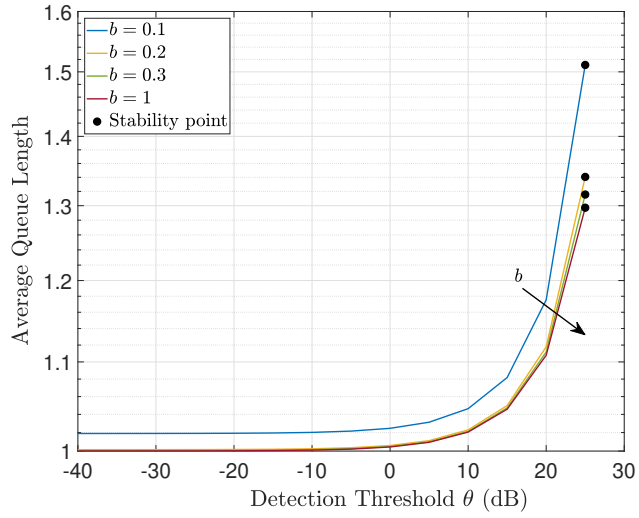

(b) $a=0.1, N_{c}=8, N=4$

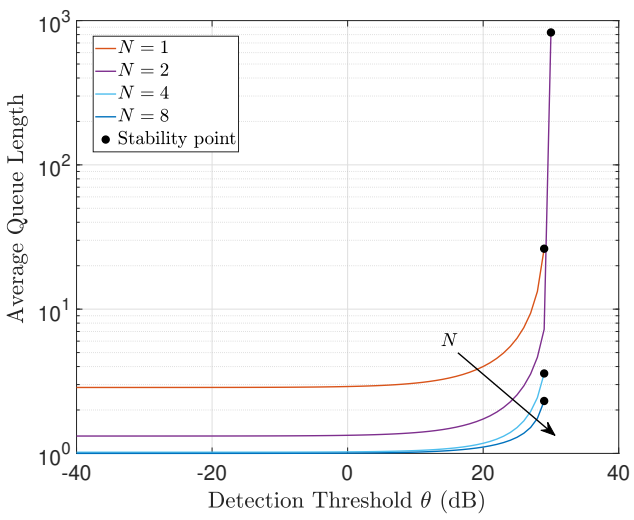

(d) $a=b=0.1, N_{c}=8$

Figure 6. Average queue length versus the detection threshold $\theta$ in $\mathrm{dB}$ for $M=8$, and $\lambda_{B}=\lambda_{D}=-20 \mathrm{~dB}$ with different packet arrival probability $a$, different BS activities, different number of channels, and different number of transmitted packets.

$$
\begin{aligned}
& \stackrel{(c)}{=} \exp \left(-2 \pi d \lambda_{D} \int_{0}^{\infty} \frac{s P_{D} r^{-\alpha_{D}}}{1+s P_{D} r^{-\alpha_{D}}} r d r\right) \\
& \stackrel{(d)}{=} \exp \left(-2 \pi d \lambda_{D}\left(s P_{D}\right)^{\frac{2}{\alpha_{D}}} \int_{0}^{\infty} \frac{u}{1+u^{\alpha_{D}}} d u\right) \\
& \stackrel{(e)}{=} \exp \left(-\frac{2 \pi^{2} d \lambda_{D} P_{D}^{\frac{2}{\alpha_{D}}}}{\alpha_{D} \sin \left(\frac{2 \pi}{\alpha_{D}}\right)} s^{\frac{2}{\alpha_{D}}}\right),
\end{aligned}
$$

where (a) is obtained due to the independence between the channels, (b) is obtained using the moment generating function (MGF) of the exponential distribution, (c) is obtained using the probability generating function (PGFL) of $\Phi_{D} \backslash y_{0}$, (d) is obtained by a change of variables $u=\frac{r}{\left(s P_{D}\right)^{\frac{1}{\alpha_{D}}}}$, and (e) is obtained using [37, (3.241.2)].

\section{APPENDIX B}

\section{Statistics of Received Power}

To derive the CDF of $P_{H}$, we first derive the LT of the aggregate downlink power in a similar way as in Appendix A as $\mathcal{L}_{C}(s)=\exp \left(-\kappa_{0} s^{\frac{2}{\alpha_{B}}}\right)$. Then, we invert $\mathcal{L}_{C}(s)$ via applying the Bromwich inversion theorem to show that the CDF of $P_{r}$ is given by

$$
\begin{aligned}
F_{P_{r}}(x) & =\frac{1}{2 i \pi} \lim _{T \rightarrow \infty} \int_{\gamma-i T}^{\gamma+i T} \frac{e^{s x}}{s} \exp \left(-\kappa_{0} s^{\frac{2}{\alpha_{B}}}\right) d s \\
& =1-\frac{1}{2 \pi i} \int_{0}^{\infty} \frac{e^{-u x}}{u}\left[-e^{-\kappa_{0} u^{\frac{2}{\alpha_{B}}} e^{\frac{2 i \pi}{\alpha_{B}}}}+e^{\left.-\kappa_{0} u^{\frac{2}{\alpha_{B}}} e^{-\frac{2 i \pi}{\alpha_{B}}}\right] d u}\right.
\end{aligned}
$$

$$
\begin{aligned}
& =1-\frac{1}{\pi} \int_{0}^{\infty} \frac{e^{-u x}}{u} \exp \left(-\kappa_{0} u^{\frac{2}{\alpha_{B}}} \cos \left(\frac{2 \pi}{\alpha_{B}}\right)\right) \\
& \times \sin \left(\kappa_{0} u^{\frac{2}{\alpha_{B}}} \sin \left(\frac{2 \pi}{\alpha_{B}}\right)\right) d u \\
& =1-\frac{\alpha_{B}}{2 \pi} \int_{0}^{\infty} \frac{e^{-x\left(\frac{t}{\kappa_{0}}\right)^{\frac{\alpha_{B}}{2}}}}{t} \exp \left(-t \cos \left(\frac{2 \pi}{\alpha_{B}}\right)\right) \sin \left(t \sin \left(\frac{2 \pi}{\alpha_{B}}\right)\right) d t
\end{aligned}
$$

For $\alpha_{B}=4$, the CDF and PDF of $P_{H}$ are deduced using [37, 3.953.6].

\section{REFERENCES}

[1] F. Benkhelifa, H. ElSawy, J. A. McCann, and M. Alouini, "Recycling cellular downlink energy for overlay self-sustainable IoT networks," in IEEE Global Communications Conference (GLOBECOM'2018), December 2018, pp. 1-7.

[2] A. Al-Fuqaha, M. Guizani, M. Mohammadi, M. Aledhari, and M. Ayyash, "Internet of Things: A survey on enabling technologies, protocols, and applications," IEEE Communications Surveys Tutorials, vol. 17, no. 4, pp. 2347-2376, Fourthquarter 2015.

[3] A. Bader, H. ElSawy, M. Gharbieh, M. S. Alouini, A. Adinoyi, and F. Alshaalan, "First mile challenges for large-scale IoT," IEEE Communications Magazine, vol. 55, no. 3, pp. 138-144, March 2017.

[4] A. Laya, C. Kalalas, F. Vazquez-Gallego, L. Alonso, and J. AlonsoZarate, "Goodbye, ALOHA!" IEEE Access, vol. 4, pp. 2029-2044, 2016.

[5] Y. P. E. Wang, X. Lin, A. Adhikary, A. Grovlen, Y. Sui, Y. Blankenship, J. Bergman, and H. S. Razaghi, "A primer on 3GPP narrowband Internet of Things," IEEE Communications Magazine, vol. 55, no. 3, pp. 117123, March 2017. 


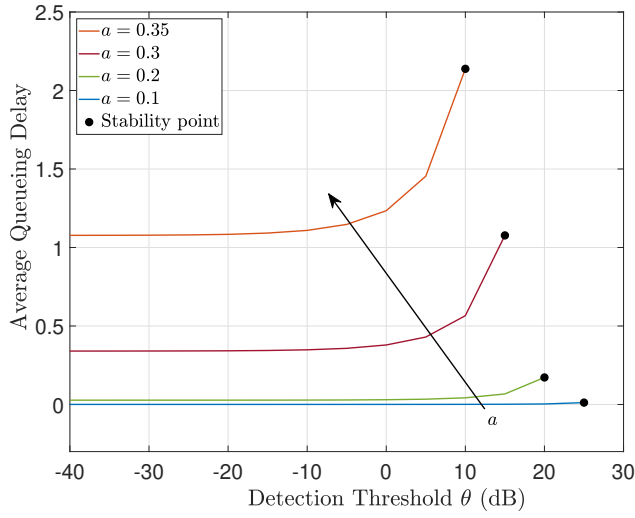

(a) $b=0.1, N_{c}=8, N=4$

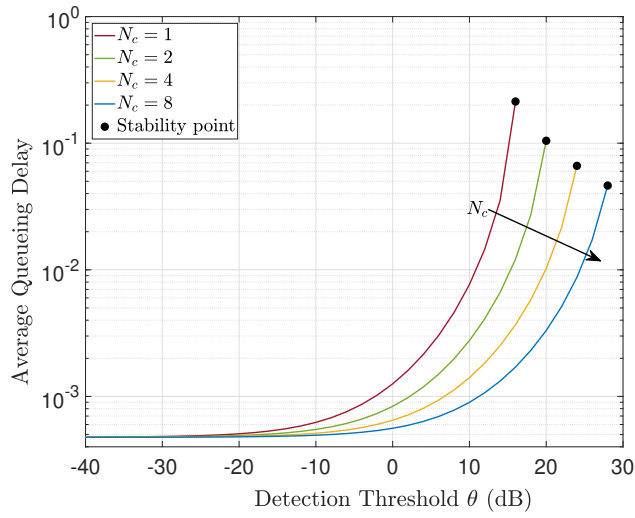

(c) $a=b=0.1, N=4$

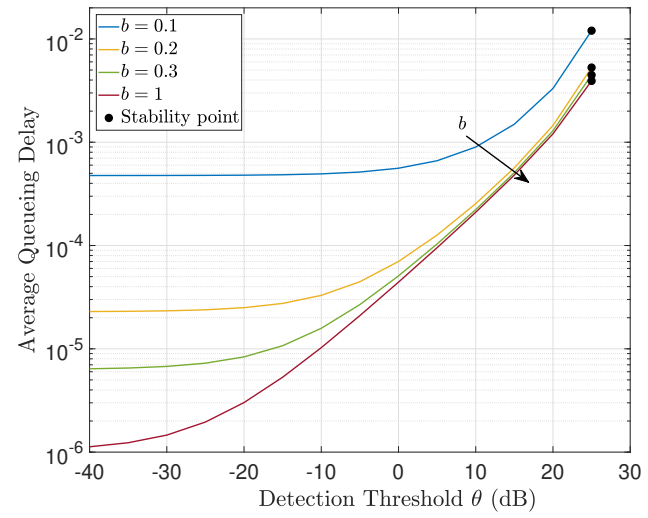

(b) $a=0.1, N_{c}=8, N=4$

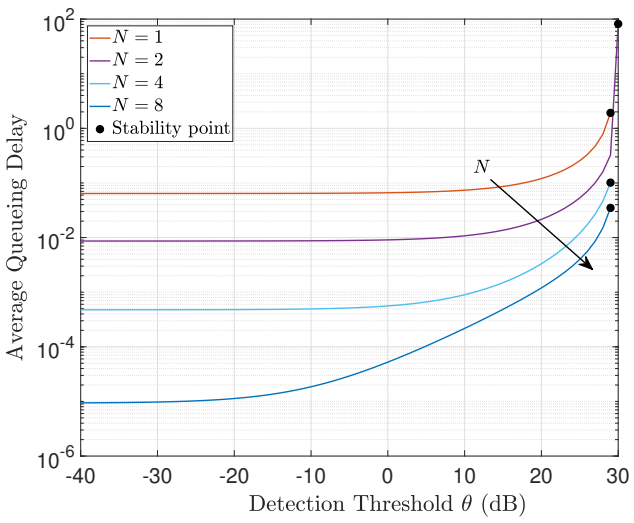

(d) $a=b=0.1, N_{c}=8$

Figure 7. Average queue delay versus the detection threshold $\theta$ in $\mathrm{dB}$ for $M=8$, and $\lambda_{B}=\lambda_{D}=-20 \mathrm{~dB}$ with different packet arrival probability $a$, different BS activities, different number of channels, and different number of transmitted packets.

[6] R. S. Sinha, Y. Wei, and S.-H. Hwang, "A survey on LPWA technology: LoRa and NB-IoT," ICT Express, vol. 3, no. 1, pp. 14 - 21, March 2017.

[7] X. Lu, P. Wang, D. Niyato, D. I. Kim, and Z. Han, "Wireless networks with RF energy harvesting: A contemporary survey," IEEE Communications Surveys Tutorials, vol. 17, no. 2, pp. 757-789, Secondquarter 2015.

[8] H. ElSawy, A. Sultan-Salem, M. S. Alouini, and M. Z. Win, "Modeling and analysis of cellular networks using stochastic geometry: A tutorial," IEEE Communications Surveys Tutorials, vol. 19, no. 1, pp. 167-203, Firstquarter 2017.

[9] N. Kouzayha, Z. Dawy, J. G. Andrews, and H. ElSawy, "Joint downlink/uplink RF wake-up solution for IoT over cellular networks," IEEE Transactions on Wireless Communications, vol. 17, no. 3, pp. 1574 1588, March 2018.

[10] Z. Qin, Y. Liu, G. Y. Li, and J. A. McCann, "Modelling and analysis of low-power wide-area networks," in IEEE International Conference on Communications (ICC'2017), May 2017, pp. 1-7.

[11] M. A. Kishk and H. S. Dhillon, "Joint uplink and downlink coverage analysis of cellular-based RF-powered IoT network," IEEE Transactions on Green Communications and Networking, vol. PP, no. 99, pp. 1-1, 2017.

[12] M. Gharbieh, H. ElSawy, A. Bader, and M. S. Alouini, "Spatiotemporal stochastic modeling of IoT enabled cellular networks: Scalability and stability analysis," IEEE Transactions on Communications, vol. 65, no. 8, pp. 3585-3600, August 2017.

[13] M. Gharbieh, H. ElSawy, H. Yang, A. Bader, and M. Alouini, "Spatiotemporal model for uplink IoT traffic: Scheduling and random access paradox," IEEE Transactions on Wireless Communications, vol. 17, no. 12, pp. 8357-8372, December 2018 .

[14] N. Jiang, Y. Deng, X. Kang, and A. Nallanathan, "Random access analysis for massive IoT networks under a new spatio-temporal model: A stochastic geometry approach," IEEE Transactions on Communications, vol. 66, no. 11, pp. 5788-5803, November 2018.

[15] Y. Zhong, T. Q. S. Quek, and X. Ge, "Heterogeneous cellular networks with spatio-temporal traffic: Delay analysis and scheduling," IEEE Journal on Selected Areas in Communications, vol. 35, no. 6, pp. 13731386, June 2017.

[16] G. Chisci, H. ElSawy, A. Conti, M. Alouini, and M. Z. Win, "Latency in downlink cellular networks with random scheduling," in IEEE International Conference on Communications (ICC'2019), May 2019, pp. $1-6$.

[17] H. H. Yang, Y. Wang, and T. Q. S. Quek, "Delay analysis of random scheduling and round robin in small cell networks," IEEE Wireless Communications Letters, vol. 7, no. 6, pp. 978-981, December 2018.

[18] G. Chisci, H. Elsawy, A. Conti, M. Alouini, and M. Z. Win, "Uncoordinated massive wireless networks: Spatiotemporal models and multiaccess strategies," IEEE/ACM Transactions on Networking, vol. 27, no. 3, pp. 918-931, June 2019.

[19] A. H. Sakr and E. Hossain, "Analysis of K-tier uplink cellular networks with ambient RF energy harvesting," IEEE J. Sel. Areas Commun., vol. 33, no. 10, pp. 2226-2238, October 2015.

[20] T. A. Khan, P. V. Orlik, K. J. Kim, R. W. Heath, and K. Sawa, "A stochastic geometry analysis of large-scale cooperative wireless networks powered by energy harvesting," IEEE Transactions on Communications, vol. 65, no. 8, pp. 3343-3358, August 2017.

[21] I. Flint, X. Lu, N. Privault, D. Niyato, and P. Wang, "Performance analysis of ambient RF energy harvesting: A stochastic geometry approach," in 2014 IEEE Global Communications Conference, December 2014, pp. $1448-1453$

[22] H. S. Dhillon, Y. Li, P. Nuggehalli, Z. Pi, and J. G. Andrews, "Fundamentals of base station availability in cellular networks with energy harvesting," in 2013 IEEE Global Communications Conference (GLOBECOM), December 2013, pp. 4110-4115.

[23] H. Inaltekin, M. Chiang, H. V. Poor, and S. B. Wicker, "On unbounded path-loss models: effects of singularity on wireless network performance," IEEE Journal on Selected Areas in Communications, vol. 27, no. 7, pp. 1078-1092, September 2009.

[24] H. Dhillon, R. Ganti, and J. Andrews, "Load-aware modeling and 
analysis of heterogeneous cellular networks," IEEE Trans. Wireless Commun., vol. 12, no. 4, pp. 1666-1677, April 2013.

[25] M. Di Renzo and W. Lu, "Stochastic geometry modeling and performance evaluation of MIMO cellular networks using the equivalentin-distribution (EiD)-based approach," IEEE Trans. Commun., vol. 63, no. 3, pp. 977-996, March 2015.

[26] K. Aly, H. ElSawy, and M. S. Alouini, "Modeling cellular networks with full duplex D2D communication: A stochastic geometry approach," IEEE Trans. Commun., vol. 64, no. 10, pp. 4409-4424, October 2016.

[27] M. Haenggi, "The meta distribution of the SIR in Poisson bipolar and cellular networks," IEEE Transactions on Wireless Communications, vol. 15, no. 4, pp. 2577-2589, April 2016.

[28] Z. Gong and M. Haenggi, "Interference and outage in mobile random networks: Expectation, distribution, and correlation," IEEE Transactions on Mobile Computing, vol. 13, no. 2, pp. 337-349, February 2014.

[29] L. Xiao, P. Wang, D. Niyato, D. Kim, and Z. Han, "Wireless networks with RF energy harvesting: A contemporary survey," IEEE Communications Surveys Tutorials, vol. PP, no. 99, pp. 1-1, 2014.

[30] B. Clerckx, R. Zhang, R. Schober, D. W. K. Ng, D. I. Kim, and H. V. Poor, "Fundamentals of wireless information and power transfer: From RF energy harvester models to signal and system designs," CoRR, vol. abs/1803.07123, 2018. [Online]. Available: http://arxiv.org/abs/1803.07123

[31] E. Boshkovska, D. W. K. Ng, N. Zlatanov, and R. Schober, "Practical non-linear energy harvesting model and resource allocation for SWIPT systems," IEEE Communications Letters, vol. 19, no. 12, pp. 2082-2085, December 2015.

[32] B. Clerckx, "Wireless information and power transfer: Nonlinearity, waveform design, and rate-energy tradeoff," IEEE Transactions on Signal Processing, vol. 66, no. 4, pp. 847-862, February 2018.

[33] L. Shi, L. Zhao, and K. Liang, "Power allocation for wireless powered MIMO transmissions with non-linear RF energy conversion models," China Communications, vol. 14, no. 2, pp. 57-64, February 2017.

[34] A. S. Alfa, Applied Discrete-Time Queues, 2nd ed. Springer Publishing Company, Incorporated, 2015.

[35] I. Krikidis, T. Charalambous, and J. S. Thompson, "Buffer-aided relay selection for cooperative diversity systems without delay constraints," IEEE Transactions on Wireless Communications, vol. 11, no. 5, pp 1957-1967, May 2012

[36] Y. Zhou and W. Zhuang, "Performance analysis of cooperative communication in decentralized wireless networks with unsaturated traffic," IEEE Trans. Wireless Commun., vol. 15, no. 5, pp. 3518-3530, May 2016.

[37] I. S. Gradshteyn and I. M. Ryzhik, Table of Integrals, Series, and Products, 5th ed. San Diego, CA: Academic, 1994. 